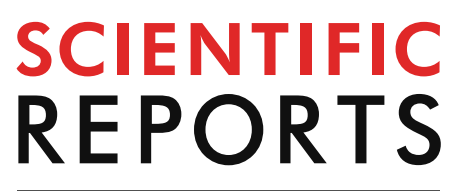

natureresearch

Check for updates

\title{
Transcriptomic determinants
} of the response of ST-111 Pseudomonas aeruginosa AG1 to ciprofloxacin identified by a top-down systems biology
approach

José Arturo Molina-Mora ${ }^{1 凶}$, Diana Chinchilla-Montero ${ }^{1}$, Maribel Chavarría-Azofeifa ${ }^{1}$, Alejandro J. Ulloa-Morales ${ }^{2}$, Rebeca Campos-Sánchez ${ }^{3}$, Rodrigo Mora-Rodríguez ${ }^{1}$, Leming $\mathrm{Shi}^{4}$ \& Fernando García ${ }^{1}$

Pseudomonas aeruginosa is an opportunistic pathogen that thrives in diverse environments and causes a variety of human infections. Pseudomonas aeruginosa AG1 (PaeAG1) is a high-risk sequence type 111 (ST-111) strain isolated from a Costa Rican hospital in 2010. PaeAG1 has both blaVIM-2 and blaIMP-18 genes encoding for metallo- $\beta$-lactamases, and it is resistant to $\beta$-lactams (including carbapenems), aminoglycosides, and fluoroquinolones. Ciprofloxacin (CIP) is an antibiotic commonly used to treat $P$. aeruginosa infections, and it is known to produce DNA damage, triggering a complex molecular response. In order to evaluate the effects of a sub-inhibitory CIP concentration on PaeAG1, growth curves using increasing CIP concentrations were compared. We then measured gene expression using RNA-Seq at three time points $(0,2.5$ and $5 \mathrm{~h}$ ) after CIP exposure to identify the transcriptomic determinants of the response (i.e. hub genes, gene clusters and enriched pathways). Changes in expression were determined using differential expression analysis and network analysis using a topdown systems biology approach. A hybrid model using database-based and co-expression analysis approaches was implemented to predict gene-gene interactions. We observed a reduction of the growth curve rate as the sub-inhibitory CIP concentrations were increased. In the transcriptomic analysis, we detected that over time CIP treatment resulted in the differential expression of 518 genes, showing a complex impact at the molecular level. The transcriptomic determinants were 14 hub genes, multiple gene clusters at different levels (associated to hub genes or as co-expression modules) and 15 enriched pathways. Down-regulation of genes implicated in several metabolism pathways, virulence elements and ribosomal activity was observed. In contrast, amino acid catabolism, RpoS factor, proteases, and phenazines genes were up-regulated. Remarkably, $>80$ resident-phage genes were up-regulated after CIP treatment, which was validated at phenomic level using a phage plaque assay. Thus, reduction of the growth curve rate and increasing phage induction was evidenced as the CIP concentrations were increased. In summary, transcriptomic and network analyses, as well as the growth curves and phage plaque assays provide evidence that PaeAG1 presents a complex, concentration-dependent response to sub-inhibitory CIP exposure, showing pleiotropic effects at the systems level. Manipulation of these determinants, such as phage genes, could be used to gain

\footnotetext{
${ }^{1}$ Centro de Investigación en Enfermedades Tropicales (CIET), Facultad de Microbiología, Universidad de Costa Rica, San José, Costa Rica. ${ }^{2}$ Chemical Genomics Centre (CGC), Max-Planck-Institute for Molecular Physiology, Dortmund, Germany. ${ }^{3}$ Centro de Investigación en Biología CelularY Molecular (CIBCM), Facultad de Microbiología, Universidad de Costa Rica, San José, Costa Rica. ${ }^{4}$ Human Phenome Institute (HuPI), Fudan University, Shanghai, China. ${ }^{\boxplus}$ email: jose.molinamora@ucr.ac.cr
} 
more insights about the regulation of responses in PaeAG1 as well as the identification of possible therapeutic targets. To our knowledge, this is the first report of the transcriptomic analysis of CIP response in a ST-111 high-risk $P$. aeruginosa strain, in particular using a top-down systems biology approach.

$\begin{array}{ll}\text { Abbreviations } \\ \text { CIP } & \text { Ciprofloxacin } \\ \text { DEGs } & \text { Differentially expressed genes } \\ \text { GGI } & \text { Gene-gene interaction } \\ \text { GSEA } & \text { Gene set enrichment analysis } \\ \text { KEGG } & \text { Kyoto encyclopedia of genes and genomes } \\ \text { MIC } & \text { Minimum inhibitory concentration } \\ \text { MLST } & \text { Multilocus sequence typing } \\ \text { OD }_{600 n m} & \text { Optical density measured at } 600 \mathrm{~nm} \\ \text { PaeAG1 } & \text { Pseudomonas aeruginosa Strain AG1 } \\ \text { PCA } & \text { Principal components analysis } \\ \text { QC } & \text { Quality control } \\ \text { RNA-Seq } & \text { RNA sequencing } \\ \text { ST-111 } & \text { Sequence type 111 } \\ \text { WGCNA } & \text { Weighted gene co-expression network analysis } \\ \text { WHO } & \text { World Health Organization (WHO) }\end{array}$

Pseudomonas aeruginosa is a ubiquitous Gram-negative organism which thrives in diverse environments and acts as an opportunistic pathogen ${ }^{1}$. The ability of this pathogen to cause a variety of human infections is facilitated by its nutritional versatility ${ }^{2}$, resistance to a wide spectrum of antibiotics, and virulence factors ${ }^{3,4}$. Pseudomonas aeruginosa AG1 (PaeAG1) is a multiresistant high-risk sequence type 111 (ST-111) strain (GenBank CP045739). It was isolated from a Costa Rican hospital and it was the first report of an isolate of P. aeruginosa carrying both blaVIM-2 and blaIMP-18 genes encoding for metallo- $\beta$-lactamases enzymes (carbapenemases), located in two independent integron $s^{5,6}$. PaeAG1 is resistant to $\beta$-lactams (including carbapenems), aminoglycosides, and fluoroquinolones, being only sensitive to colistin. In addition to this multidrug-resistant feature, as in other $P$. aeruginosa strains, the ability to colonize nosocomial environments makes this strain a high-risk clone ${ }^{7}$. Owed to this antibiotic resistance profile, including resistance to carbapenems, PaeAG1 is classified as a Priority 1 (critical) organism according to the World Health Organization (WHO) $)^{8}$.

Antibiotic resistance is a major threat to public health because it compromises the administration of appropriate antibiotic therapy, and reduces the therapeutic options to treat infections, increasing patient morbidity and mortality ${ }^{9,10}$. This situation is aggravated by the emergence of strains resistant to multiple antibiotics ${ }^{11}$, limitation of the knowledge of interactions with pathogens and mechanisms of action of antimicrobial agents, and development of new antibiotics ${ }^{12}$. Use of antibiotics below the minimum inhibitory concentration (MIC) or sub-inhibitory concentrations also contributes to antibiotic resistance as they allow strains to continue growing and can select for pre-existing resistant organisms ${ }^{13}$. Since sub-inhibitory antibiotic concentrations are found in many natural environments, bacteria can naturally trigger mechanisms of tolerance ${ }^{14}$. However, the fundamental mechanisms of bacterial tolerance to antibiotics have not been fully elucidated ${ }^{15}$.

It has been shown that the perturbation induced by many antibiotics leads to stress conditions in prokaryotic cells ${ }^{16}$, which can induce DNA damage ${ }^{17}$. Stressors activate the regulation of gene expression or the activity and stability of existing proteins to induce adaptation mechanisms ${ }^{16}$. Organisms have evolved numerous DNA repair pathways to eliminate DNA damage and restart DNA replication ${ }^{18}$. Regulatory networks of transcriptional responses to DNA damage involves not only DNA repair enzymes, but also diverse proteins with roles in cell division, metabolism modulation, genetic rearrangements and exchange, mutation, and virulence factor production ${ }^{19}$.

Ciprofloxacin (CIP) is a fluoroquinolone antibiotic used to treat P. aeruginosa infection $\mathrm{s}^{20}$. CIP is well-known to produce DNA damage by inhibiting DNA gyrase and topoisomerase IV, leading to DNA strand breaks ${ }^{21}$. Mutations in these genes are responsible for CIP resistance by losing drug affinity ${ }^{22}$. CIP has been used to study stress responses in this bacterial group ${ }^{12,23}$, in particular with the induction of the SOS response as a mechanism of DNA damage repair ${ }^{17,24,25}$. In P. aeruginosa, the SOS response regulon is composed of 15 genes, including rec $A$ and lexA genes ${ }^{26}$. Upon DNA damage, RecA recognizes the single-stranded DNA (ssDNA) forming filaments and induces the autocleavage of the repressor LexA. This response leads to the expression of genes related to DNA damage repair ${ }^{27}$. Other LexA-like repressors are regulated during SOS activation, including elements of phages and pyocines ${ }^{19}$. SOS also mediates responses to resistance element transfer, generation of mutations and evolution of resistance ${ }^{26}$, as well as appearance of persister cells ${ }^{24}$.

However, modulation of stress responses after DNA damage is not limited to SOS response. RpoS is a general stress sigma factor $(\sigma S)$ known as a central element in a regulatory network that governs the expression of stationary-phase-induced genes ${ }^{28}$ to maintain cell viability ${ }^{29}$. This regulator is strongly induced when cells are exposed to various stress conditions, including antibiotics, $\mathrm{pH}$ downshift, starvation, and hyperosmolarity ${ }^{30}$. RpoS regulates more than 50 genes in Pseudomonas aeruginos $a^{31}$, including virulence factors ${ }^{32}$.

The SOS and RpoS regulons are complementary mechanisms in response to certain stresses and that protect bacteria from DNA damage ${ }^{33}$. Lon protease ${ }^{11}$ and $\mathrm{AmpR}^{34}$ can modulate both SOS and RpoS regulons. In addition, both responses can regulate key genes such as $p o l B^{18}$, iraD $D^{19}$, and $\operatorname{din} B^{33}$. The connection between RpoS and SOS responses seems to be associated with a mechanism to maximize survival and fitness of cells, and to 
maintain genome stability ${ }^{18}$. These responses can modulate virulence factors (including quorum sensing and biofilm formation), and increase homologous recombination and mutation frequencies ${ }^{33,35}$. However, other SOS and RpoS independent mechanisms are also known to be present in bacteria ${ }^{36}$, including P. aeruginosa after CIP treatment ${ }^{12,26}$ with variable results depending on strains and showing a mosaic response ${ }^{12}$.

Although the full mechanisms of all these molecular responses are not well understood, it is known that cells respond to stress conditions by complex regulatory systems that control gene expression ${ }^{37}$. Since a key objective in biological research is to describe molecular interactions ${ }^{38}$, the use of networks analysis is a common approach to describe complex biological systems and to mathematically model gene-gene interactions (GGI) with graphical representations (genes as nodes and interactions as edges) ${ }^{39}$. Molecules are thereby studied not only at a single level, but emergent properties are identified to describe and understand the complexity of the gene networking response and the emergent properties towards the stress condition. Functional status of genes by a top-down systems biology perspective, starting from "whole"-omics data to identify specific determinants or elements of biological importance, can be evaluated by construction of large scale networks ${ }^{40}$. For this purpose, data analysis from high-throughput technologies such as microarrays and RNA sequencing (RNA-Seq) can be used to describe molecular interactions at transcriptomic level ${ }^{38,41}$. Thus, to understand or to infer mechanisms associated with the transcriptional response, it is possible to build gene regulatory networks either using databases or based on co-expression data ${ }^{39,42,43}$. These networks allow to gain insight into response to stress conditions ${ }^{44}$, leading to the identification of gene clusters or even hub genes as candidate biomarkers or modulators with potential to become key therapeutic targets ${ }^{43,45}$.

In $P$. aeruginosa, rapid adaptation to stress conditions is partially explained by the modulation of the global gene expression, which represents around $8 \%$ of all coding genes ${ }^{3}$. This regulation induces pleiotropic effects on its genomic regulatory network ${ }^{46}$, as previously shown using systems biology ${ }^{47}$, and the transcriptomic profiling of the response to $\mathrm{CIP}^{12,26,48}$.

In this work we first evaluated PaeAG1 growth at sub-inhibitory CIP concentrations, showing growth reduction as CIP was increased. We hypothesized that after exposing PaeAG1 to ciprofloxacin, even at sub-inhibitory concentrations, transcriptomic determinants will be triggered, including bacterial growth modulators. Thus, the aim was to identify transcriptomic determinants associated with the response to CIP in PaeAG1 using RNA-Seq profiling and network analysis by a top-down systems biology approach. Results showed that PaeAG1 generates a complex response to CIP exposure, evidencing pleiotropic effects involving the regulation of multiple hub genes, gene clusters and enriched pathways (transcriptomic determinants), many of them related to growth. As evidenced at the transcriptomic and the phenomic levels, phage induction was a particular trait modulated by $\mathrm{CIP}$ in a concentration-dependent manner with a correlation with bacterial growth reduction.

\section{Methods}

The general pipeline followed in this study to identify the transcriptomic determinants associated with the response to CIP in PaeAG1 is shown in Fig. 1.

Bacterial isolate. The PaeAG1 strain is a Costa Rican multiresistant isolate from a sputum sample of a patient with pneumonia at the Intensive Care Unit of the San Juan de Dios Hospital (San José, Costa Rica) ${ }^{6}$. PaeAG1 exhibits resistance to $\beta$-lactams (including carbapenems, $\mathrm{MIC}_{\text {Meropenem }} 32 \mu \mathrm{g} / \mathrm{mL}$ and $\mathrm{MIC}_{\text {Imipenem }}>32 \mu \mathrm{g} / \mathrm{mL}$ ), aminoglycosides $\left(\mathrm{MIC}_{\text {Gentamycin }} 128 \mu \mathrm{g} / \mathrm{mL}\right.$ and $\mathrm{MIC}_{\text {Tobramycin }}>192 \mu \mathrm{g} / \mathrm{mL}$ ) and fluoroquinolones $\left(\mathrm{MIC}_{\text {Ciprofloxacin }}\right.$ $32 \mu \mathrm{g} / \mathrm{mL}$ ), and it is only sensitive to colistin ( $\mathrm{MIC}_{\text {Colistin }} 2 \mu \mathrm{g} / \mathrm{mL}$ ). We recently assembled and annotated the PaeAG1 genome ${ }^{5}$, and genome sequence and annotation are available in Genbank under accession CP045739 (Bioproject PRJNA587210).

Growth curves assay. Three independent cultures of PaeAG1 cells were grown to exponential-phase overnight in Lysogenic Broth (LB) at $37^{\circ} \mathrm{C}$ with shaking (pre-culture to reach mid-log phase). Then, five aliquots were added to $50 \mathrm{~mL}$ of fresh $\mathrm{LB}$ broth to an initial optical density measured at $600 \mathrm{~nm}\left(\mathrm{OD}_{600 \mathrm{~nm}}\right)$ of 0.01 . Each sample was treated with a specific CIP concentration of 0.0 (control), 5.0, 12.5, 25.0 or $50.0 \mu \mathrm{g} / \mathrm{mL}$ (final concentrations). Growth of cultures was monitored by $\mathrm{OD}_{600 \mathrm{~nm}}$ at times $0,2,4,6,8,12$ and $16 \mathrm{~h}$. Comparison of different CIP concentrations was done by assessing growth curve kinetics, including lag and exponential phases. As a complementary assay, evaluation of two other antibiotics was done in exactly the same growth conditions, but antibiotic concentrations depended on the MIC: imipenem (carbapenem) and tobramycin (aminoglycoside). See results and supplementary Figure S1 for details.

The growth curves were statistically compared to the control growth curve using a two-way ANOVA with Bonferroni post-tests (significance level of 95\%), similar to ${ }^{49}$, using the time and concentrations as factors. We also ran a unpaired t-test (95\% significance) comparing area under curve (AUC) of each growth curve against the control, similar to ${ }^{50}$. Analyses were done using Prism (GraphPad Software, Inc., La Jolla, CA). To perform the transcriptomic assay, we used the results from growth curves to select a specific sub-inhibitory CIP concentration at which there were no major changes in the growth rate after treatment.

RNA isolation and RNA sequencing. In order to evaluate the molecular response of PaeAG1 to a subinhibitory CIP concentration, a transcriptomic assay was designed using RNA-Seq technology, as described below.

Growth conditions. PaeAG1 cells were grown under the same conditions as detailed before but treatment was done using a single CIP concentration of $12.5 \mu \mathrm{g} / \mathrm{mL}$ (see "Results" for details of concentration selection). Immediately after adding treatment, an aliquot was taken as control (time $0 \mathrm{~h}$ ), and cells were kept growing for 2.5 and 


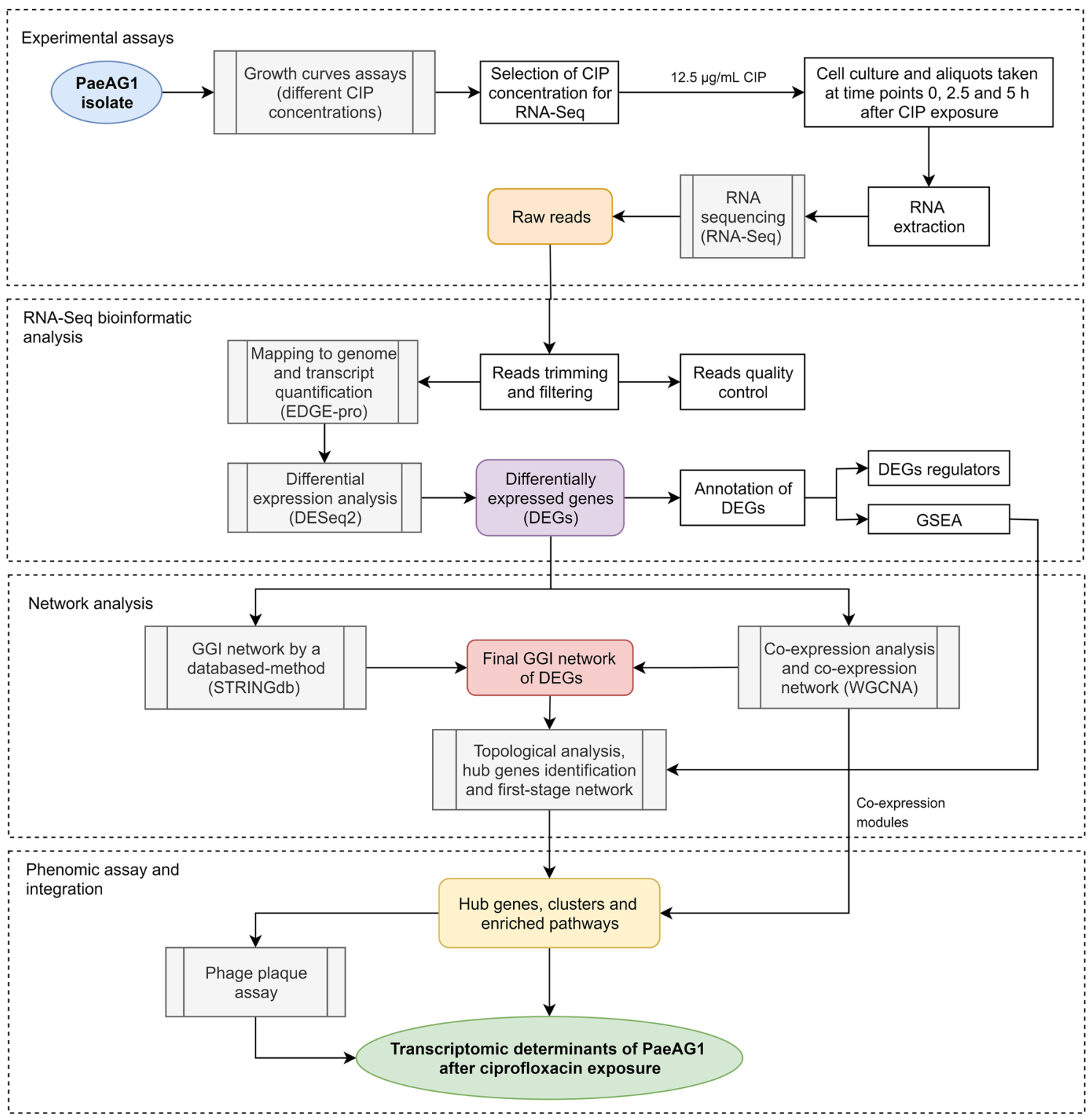

Figure 1. General pipeline to identify the transcriptomic determinants of the response of $P$. aeruginosa AG1 to ciprofloxacin (CIP). After growth curves assessment, a specific CIP concentration was used to sequence RNA (RNA-Seq) at 0, 2.5 and $5 \mathrm{~h}$ after exposure. DEGs were identified and used to build GGI networks. Transcriptomic determinants were identified by network analysis. Findings were verified at phenomic level using a phage plaque assay.

$5 \mathrm{~h}$ (times were selected according to preliminary results of phage induction, see "Methods" for Phage plaque assays). This was done with three independent cultures for a total of nine aliquots, three replicates per time.

RNA isolation. Aliquots from the cultures were preserved in two volumes of RNA protect reagent (QIAGEN) and cells were stored at $4{ }^{\circ} \mathrm{C}$ until RNA extraction. At the end of the sample collection period, total RNA was extracted using the RNeasy Mini kit (QIAGEN, UK) following the manufacturer's instructions. RiboZero Gold (Epicentre) was used to deplete bacterial rRNA from total RNA samples according to manufacturer's instructions. The quality and quantity of extracted RNA was determined using a Nanodrop (Nanodrop 2000, Thermo Scientific, UK). The RNA integrity was analyzed using Agilent 2,100 Bioanalyzer (Agilent Technologies, USA) to obtain the RNA integrity number (RIN) for all samples.

RNA sequencing. For RNA sequencing, TruSeq Stranded Total RNA library preparation kit (Illumina, USA) was used to generate cDNA (amplification with 13 PCR cycles) and libraries for $2 \times 51$ bp paired-end reads. Libraries were prepared and sequenced at the Genome Technology Center, New York University (New York, USA) on the Illumina HiSeq 2,500 platform. Sequencing generated more than $120 \mathrm{~Gb}$ of sequences ( $>300$ millions of reads in total) for all samples. 
RNA-Seq data analysis. With the aim of quantifying transcripts and identifying DEGs in PaeAG1 after CIP treatment, RNA-Seq data was analyzed including a quality control step, reads mapping to genome for transcript quantification and differential expression analysis.

Quality control (QC). QC was done before and after trimming/filtering. Reads were trimmed using Trimmomatic $\mathrm{v} 0.38^{51}$ to discard sequences with per base phred sequence quality score $<30$ and 35 minimum length. Reads were filtered using BBDuk (https://jgi.doe.gov/data-and-tools/bb-tools/) to remove adapters and reads mapping to rRNA. Sequence files were evaluated using FastQC v0.11.7 $7^{52}$ to obtain general quality control metrics. To evaluate the origin of reads sequences, FastQ-Screen ${ }^{53}$ was used to quantify the proportion of reads that mapped to reference genomes (human, mouse, and adapters contaminants, included by default) and prokaryotic sequences specifically added for this work (PaeAG1 and E. coli genomes, and rRNA 16S and 23S databases). Reports were merged using MultiQC ${ }^{54}$ to summarize all individual results. After selection, sequences for each of the nine samples had an average output of approximately 60 million reads.

Reads mapping and transcript quantification. We used EDGE-pro v1.3.1 software to: map RNA-Seq reads to the PaeAG1 genome (Genbank CP045739), filter out multialigned reads, and estimate expression levels of each gene by counts ${ }^{55}$. This program was run with the default parameters, using Bowtie $2^{56}$ as read alignment algorithm. The script "edgeToDeseq.perl", provided with the software, was used to convert raw counts (EDGE-pro output) to a count-table format for further differential expression analysis. Quality control of alignments per sample was done using: Qualimap RNA-Seq tool ${ }^{57}$ to assess mapping quality, and RSeQC package ${ }^{58}$ to estimate transcripts coverage uniformity (gene body coverage) and transcript integrity number (TIN). Required formats of genome annotation files for these analyses are available in https://github.com/josemolina6/PaeAG1_genome.

Differential expression analysis. We used raw counts of transcripts to estimate differential expression. For this purpose, DESeq2 package v1.26.0 $0^{59}$ in R program v3.5. ${ }^{60}$ was used based on the negative binomial generalized linear models, using default settings. DESeq2 based normalization, absolute expression comparisons by the regularized log transformation ( $r l o g$ ), Principal Component Analysis (PCA), counts dispersion plots and clustering analysis were run in the same program. Triplicates of each time after PaeAG1 exposure to CIP were considered as a factor level. Differential expression analysis was done comparing $2.5 \mathrm{~h}$ or $5 \mathrm{~h}$ data against the initial time point at $0 \mathrm{~h}$. Hypothesis testing to select differentially expressed genes (DEGs) was done using Benjamini-Hochberg adjustment (to control false discovery rate, FDR) and $\log _{2}$ [FoldChange] (logFC) of transformed and normalized mean counts. Genes were considered up-regulated if $\log \mathrm{FC}>1$ or down-regulated if $\log \mathrm{FC}<-1$, considering an adjusted $p$-value $<0.05$ for both cases. Gene list comparisons by Venn diagrams were performed using the Draw Venn Diagram Tool (https://bioinformatics.psb.ugent.be/webtools/Venn/).

Annotation of differentially expressed genes. DEGs annotation was retrieved from our previous work $^{5}$ for the assembly and annotation of PaeAG1 genome (Genbank CP045739). Particular features per gene (including molecular function, product, gene size and domains, and sub cellular location of proteins) were explored in more detail from Pseudomonas Genome Database (https://www.pseudomonas.com/ $/)^{61}$. In addition, general regulators of the DEGs were investigated using PseudomonasNet tool (https://www.inetbio.org/pseud omonasnet/Network_regulon_form.php) with a $p$-value $<0.05$ in a context-centric analysis. Using the same platform, it was possible to identify the DEGs and their regulators that corresponded to transcription factors genes.

Analysis of DNA-protein interactions. For selected genes, protein-DNA binding sites were investigated. The CollectTF database (https://www.collectf.org/) was primarily used to search for consensus DNA binding sequences of the protein of interest and to identify modulated genes. If no information was available, promoter consensus sequences were searched from particular studies and the identification of binding sites was done using the motif-based sequence analysis tool (MEME, using Find Individual Motif Occurrences FIMO, https://meme-suite.org/tools/fimo).

In order to identify DEGs as molecular determinants (hub genes, gene clusters and key pathways) of the response to CIP in PaeAG1, a large scale gene-gene interaction (GGI) network of DEGs was built using a topdown systems biology approach. Connections between genes were predicted using two independent methods, one using a database-based model and another from co-expression analysis, detailed as follows.

Database-based method for gene-gene interactions prediction and network construction. With the aim of obtaining a high confidence GGI between DEGs using a database-based method, the Search Tool for the Retrieval of Interacting Genes database (STRINGdb) ${ }^{62}$ was used to construct a large scale GGI network for the DEGs using default parameters. All DEGs at any of the two times were used to build the main network. The resulting graph was exported and then visualized and topologically analyzed using Cytoscape software $^{63}$.

Co-expression analysis and co-expression network construction. To incorporate more interactions between DEGs, a data-driven systems biology approach was implemented using co-expression analysis with all the normalized counts of DEGs, as in recent studies ${ }^{45,64-66}$.

Modules identification using co-expression analysis. Weighted gene co-expression network analysis (WGCNA) package $^{43}$ was run in R software. Briefly, a matrix of Pearson correlation between all pairs of genes was calcu- 
lated. The adjacency matrix was then constructed using a power of $\beta=9$ as a saturation level for a soft threshold of the correlation matrix based on the criterion of scale-free topology. The topological overlap matrix was calculated. Hierarchical clustering was used to generate a dendrogram to group highly co-expressed genes, creating gene clusters called modules (arbitrarily represented by colors) using the default dynamic tree cut algorithm. Default colors given to modules were kept.

Association of co-expression modules and traits. A t-test evaluated the association between the modules (using module eigengene ME, the first principal component gene of module expression matrix) and traits of PaeAG1 according to the experimental design. For this, the times (the experiment factors $0,2.5$ and $5 \mathrm{~h}$ ) and data of phage induction at 2.5 and $5 \mathrm{~h}$ after $12.5 \mu \mathrm{g} / \mathrm{mL}$ CIP exposure were incorporated as traits (see "Phage plaque assay" section in "Methods").

Co-expression network. To visualize the whole network including the modules by colors, the WGCNA "exportNetworkToCytoscape" function was run, using a correlation threshold of 0.985 and weight = false to build an un-weighted graph of highly connected genes with very strict correlation. The data-driven graph was visualized using Cytoscape.

Integrated DEGs network construction. The final GGI network of DEGs was constructed joining the files of the well-known interactions predicted by STRING database and the strict data-driven interactions obtained from co-expression analysis (un-weighted graph). The definitive graph was visualized using Cytoscape software. Topological metrics of the graph were obtained using the defaults apps available in Cytoscape.

Enrichment analysis. For the gene set enrichment analysis (GSEA), STRINGdb was used to identify significantly enriched pathways according to KEGG database, using a cutoff of FDR $<0.05$. This analysis was run for complete gene lists of DEGs at 2.5 h, DEGs at $5 \mathrm{~h}$, and genes of each co-expression module. Results of enrichment were incorporated into the DEGs network using the Cytoscape app Omics Visualizer (https://apps.cytos cape.org/apps/omicsvisualizer).

Hub genes identification. In order to identify central or hub genes in the DEGs network of PaeAG1 after exposure to CIP, cytoHubba app ${ }^{67}$ was run in Cytoscape. To address this, bottleneck and betweenness methods were implemented with default parameters. The top 10 nodes (genes) were selected for each method using calculated metrics. All selected genes in any of the methods were labeled as hub genes. In addition, cytoHubba was also used to build two subnetworks using the hub genes, one with the selected elements only, and another including the first-stage nodes (in direct connection with hub genes) to identify gene clusters. KEGG annotation information was kept from the DEGs network.

Expression profiles of hub genes were compared to expression levels obtained in other representative studies, including the following stressors: $\mathrm{Cu}$ (copper) ${ }^{68}$, CIP (ciprofloxacin) ${ }^{26}, \mathrm{COL}$ (colistin) ${ }^{69}, \mathrm{AZM}$ (Azithromycin $)^{70}$ and $\mathrm{H}_{2} \mathrm{O}_{2}$ (hydrogen peroxide) ${ }^{71}$. Comparison was done using the general information of expression levels (down, up or variable regulation).

Phage plaques assay (validation assay at the phenomic level). To validate the transcriptomic results which showed an up-regulation of phage genes in PaeAG1 after exposure to CIP, we implemented a phage plaques assay and performed this assay in triplicate. To assess the CIP effect on phage induction, different CIP concentrations were evaluated. Evaluation was also done for imipenem and tobramycin as supplementary assays. Growth conditions were the same as described in the "Growth curve assays", until the addition of different antibiotic concentrations. At this point, cultures were kept growing for five hours and phages were isolated and quantified for each sample. During standardization, it was determined that five hours after CIP exposure was the minimum time for clear detection of phage plaques (see supplementary Figure S1-B for details). Phage plaque counts at $2.5 \mathrm{~h}$ and $5 \mathrm{~h}$ for $12.5 \mu \mathrm{g} / \mathrm{mL}$ CIP were used to associate the phage induction with co-expression modules (detailed in "Co-expression analysis" section).

Phages isolation. Protocols of ${ }^{72}$ and ${ }^{73}$ were adapted. Briefly, the culture was centrifuged for $20 \mathrm{~min}$ at $4,000 \mathrm{rpm}$, $40 \mathrm{~mL}$ of the supernatant was taken and $1 \mathrm{~mL}$ of chloroform was added to residual bacterial cells. After overnight incubation, cell debris was removed by centrifugation for $20 \mathrm{~min}$ at 3,000 rpm. The supernatant was filtered through a $0.45 \mu \mathrm{m}$ filter to select phages. A volume of $30 \mathrm{~mL}$ of the filtered supernatant was mixed with $7.5 \mathrm{~mL}$ of polyethylene glycol $(20 \%)$ and $\mathrm{NaCl}(2.5 \mathrm{M})$ to precipitate the phages. After overnight incubation, the sample was centrifuged for $30 \mathrm{~min}$ at 4,000 rpm, the supernatant was discarded and the pellet was resuspended in $250 \mu \mathrm{L}$ of phage buffer $\left(10 \mathrm{mM} \mathrm{MgSO}_{4}, 10 \mathrm{mM}\right.$ Tris- $\mathrm{HCl}$ and $\left.150 \mathrm{mM} \mathrm{NaCl}\right)$.

Phages quantification. Phages were quantified by means of Plaque Forming Units (PFU) using P. aeruginosa PAO1 as host cells. The numbers of PFU was determined using the double-agar-layer method ${ }^{74}$. Briefly, medium was composed of two agar layers, a first layer $1.5 \%$ and another to $0.5 \%$ agar concentration. P. aeruginosa PAO1 and phages were added on the second layer and phage plaques were visualized after incubation for $24 \mathrm{~h}$ at $25^{\circ} \mathrm{C}$.

An exponential regression between the CIP concentrations and the PFU was run to associate the effect of CIP exposure on the phage induction. 


\section{Bacterial growth curve}

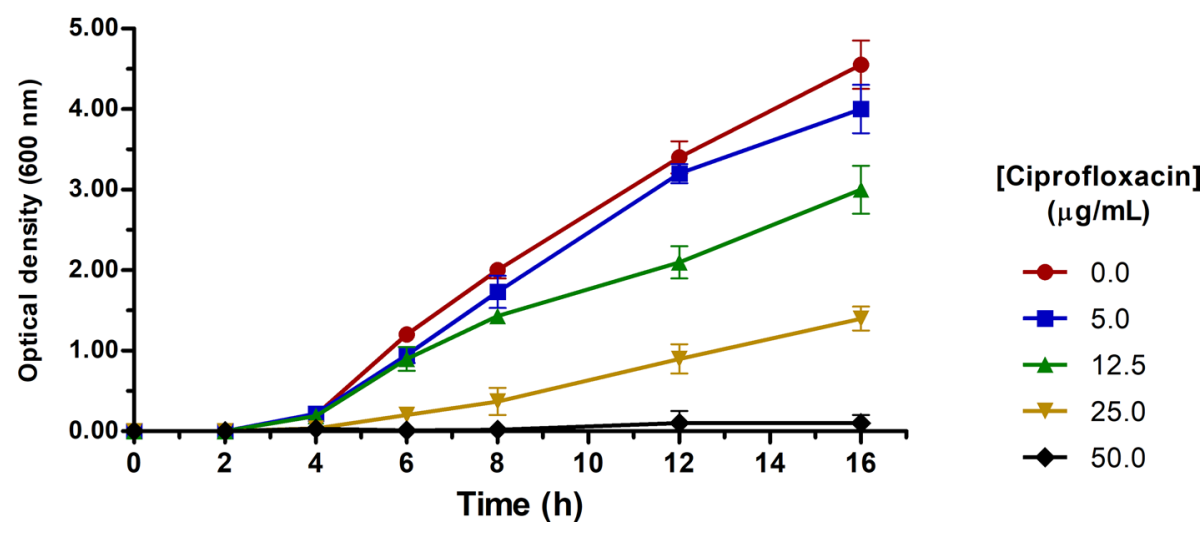

Figure 2. In vitro effects of ciprofloxacin on growth curve of PaeAG1. A growth rate reduction was observed as the CIP concentration was incremented. Area under curve (AUC) was compared using t-test $(\mathrm{p}<0.05)$, showing a statistical difference between all curves when compared to control $(0.0 \mathrm{mg} / \mathrm{mL})$. In a similar manner, two-way ANOVA found differences in the $\mathrm{OD}_{600 \mathrm{~nm}}$ and time for each case.

Ethical considerations. No animals or human participants were included in this study. Both the scientific committee of the Centro de Investigación en Enfermedades Tropicales (CIET) and Vicerrectoría de Investigación of Universidad de Costa Rica approved the study and the access to the PaeAG1 strain from the CIET collection of bacterial specimens.

\section{Results}

Concentration-dependent effect of CIP compromises the growth rate of PaeAG1. To evaluate the effects of CIP in the growth rate of PaeAG1, increasing concentrations of the antibiotic were added to exponential-phase PaeAG1, and growth was monitored over time for $16 \mathrm{~h}$. As shown in Fig. 2, OD $600 \mathrm{~nm}$ values were highly consistent between replicates (error bars represent standard deviation). All CIP curves showed a statistical significant difference on $\mathrm{OD}_{600 \mathrm{~nm}}$ compared to control ( $\mathrm{p}<0.05$ for both AUC and two-way ANOVA). Lag phase for the control and two lower CIP concentrations ( 5 and $12.5 \mu \mathrm{g} / \mathrm{mL}$ ) lasted approximately $4 \mathrm{~h}$, while the higher CIP concentration of $25.0 \mu \mathrm{g} / \mathrm{mL}$ showed a lag phase of $8 \mathrm{~h}$.

Kinetics at the exponential phase showed more variable results. There was a decrease in cell growth for $12.5 \mu \mathrm{g} / \mathrm{mL}$ CIP from $12 \mathrm{~h}$ onwards in comparison to 0 or $5.0 \mu \mathrm{g} / \mathrm{mL}$, and more evident at same time for $25 \mu \mathrm{g} /$ $\mathrm{mL}$. For the case of $50.0 \mu \mathrm{g} / \mathrm{mL}$ (higher than MIC), the growth was drastically impaired and no exponential growth was observed. These results indicate that higher CIP concentrations have a stronger effect on the growth rate, even for sub-inhibitory concentrations $\left(\mathrm{MIC}_{\text {Ciprofloxacin }} 32 \mu \mathrm{g} / \mathrm{mL}\right.$ ). Evaluation of the growth effects of other two antibiotics (imipenem and tobramycin) was also performed (supplementary Figure S1C-E, left). Unlike CIP, both cases showed no changes in the growth curves with different sub-inhibitory concentrations.

Due to the significant changes in growth curves with CIP (with respect to control) and considering a condition with enough cell mass for RNA-Seq analysis, $12.5 \mu \mathrm{g} / \mathrm{mL}$ CIP was used to evaluate the transcriptomic response of PaeAG1 to a sub-inhibitory concentration of the antibiotic.

RNA-Seq analysis identifies 518 DEGs in PaeAG1 over time after exposure to CIP. A transcriptomic analysis was conducted to evaluate the molecular response to sub-inhibitory CIP concentration in PaeAG1. To this end, samples were taken at 0 (control), 2.5 and $5 \mathrm{~h}$ after CIP treatment. To ensure exponential growth at these times, the growth curve was monitored using $\mathrm{OD}_{600 \mathrm{~nm}}$ measurements (successfully reproduced as Fig. 2), in addition to counting of Colony Forming Units (CFU), as shown in supplementary Figure S1A. After RNA was extracted, RNA integrity RIN $>9$ was obtained for all samples and paired-end RNA sequencing was performed. For all samples, quality control of raw sequence data showed good results in terms of mean quality (>30), no adapters, and no reads mapping to rRNA after filtering. Read mapping quality control showed that 98.6\% were mapped to the PaeAG1 genome, with expected uniform coverage for gene body, and TIN $>90$ for all samples. Details of assessment of transcriptomic data (counts per gene) is shown in supplementary Figure S2.

Identification of DEGs was conducted by comparing times 2.5 or $5 \mathrm{~h}$ against the initial $0 \mathrm{~h}$ time after CIP exposure (Fig. 3A,B). As shown in Table 1, 355 DEGs were identified at time 2.5 h, with 204 (57.5\%) up-regulated and 151 (42.5\%) down-regulated. At 5 h, 248 (56.6\%) genes were up-regulated, meanwhile 190 (43.4\%) were found to be down-regulated, for a total of 438 DEGs.

A total of 518 DEGs were found at any time points (union $U$ ), as shown in Fig. 3C and Table 1. These represent around $7 \%$ of the genes of PaeAG1. In addition, as presented in Fig. 3D, a total of 85 DEGs (at any time) belong to phages (27.6\% of the 308 phage genes identified in the PaeAG1 genome), most of them up-regulated as shown in Table 2, Fig. 4 and supplementary Figure S3. The phages regulated include phiCTX, F10, JBD44 and JDO24 for which 3, 10, 65 and 7 DEGs were respectively observed at any time (Table 2). 
A

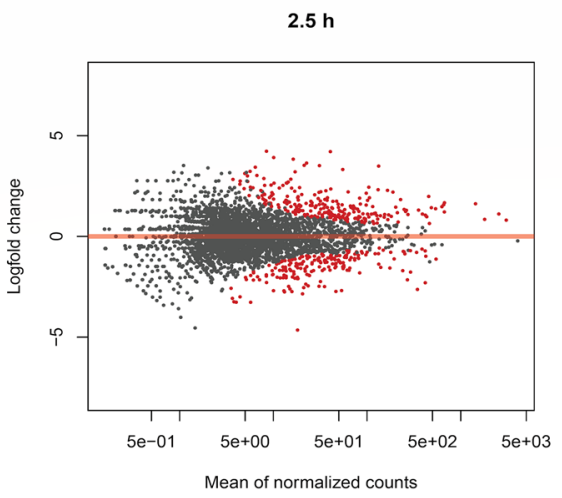

C

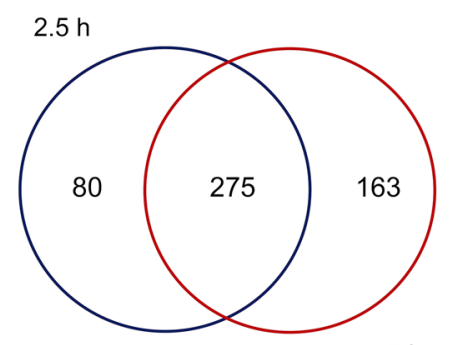

$5 \mathrm{~h}$
B

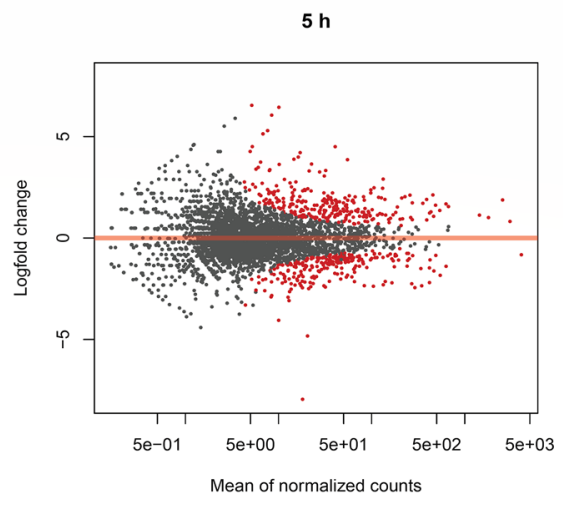

D

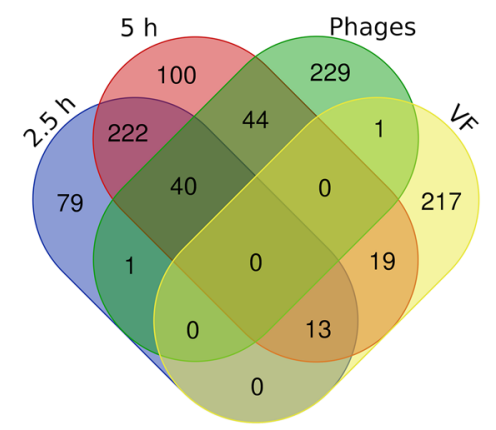

E

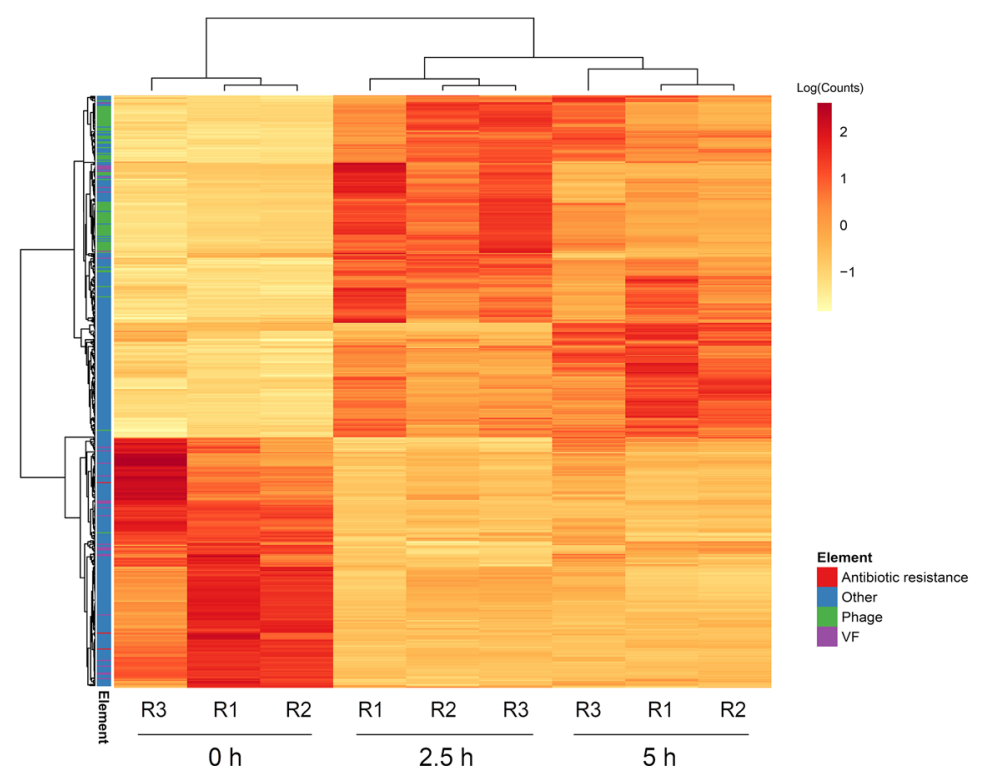

Figure 3. Differential expression analysis in PaeAG1 exposed to ciprofloxacin compared to initial time $0 \mathrm{~h}$. Selection of DEGs according to adjusted $p$-value $(\mathrm{p}<0.05)$ and $\log \mathrm{FC}(\log \mathrm{FC}<-1$ or $\log \mathrm{FC}>1)$ at $2.5 \mathrm{~h}(\mathbf{A})$ or $5 \mathrm{~h}(\mathbf{B})$ post-exposure to antibiotic. (C) Venn diagram showing the comparison of DEGs in the two evaluated times, with 275 shared genes (intersection) and total 518 genes at any time (union) with respect to time $0 \mathrm{~h}$ (control). More details in Table 1. (D) Venn diagram showing the comparison of DEGs and phage genes or virulence factors (more details in Table 2). (E) Heatmap of normalized counts and gene clustering of the total 518 DEGs at the three evaluated time points. 


\begin{tabular}{|c|c|c|c|c|}
\hline \multirow[b]{2}{*}{ DEGs } & \multicolumn{4}{|l|}{ Sets } \\
\hline & $2.5 \mathrm{~h}$ & $5 \mathrm{~h}$ & $2.5 \mathrm{~h} \cap 5 \mathrm{~h}$ & $2.5 \mathrm{~h} \cup 5 \mathrm{~h}$ \\
\hline Up regulated genes & 204 & 248 & 153 & 299 \\
\hline Down regulated genes & 151 & 190 & 118 & 223 \\
\hline Total DEGs & 355 & 438 & 275 & 518 \\
\hline
\end{tabular}

Table 1. Comparison of DEGs of PaeAG1 at 2.5 and $5 \mathrm{~h}$ after treatment with Ciprofloxacin, including counts of down or up regulated genes, shared genes (intersection) and total genes at both times (union).

\begin{tabular}{|c|c|c|c|c|c|c|c|}
\hline \multicolumn{3}{|l|}{ Determinants } & \multicolumn{5}{|c|}{ Sets of DEGs } \\
\hline Type & Specific elements & $\begin{array}{l}\text { Total genes (in } \\
\text { PaeAG1 genome) }\end{array}$ & $2.5 \mathrm{~h}$ & $5 \mathrm{~h}$ & $2.5 \mathrm{~h} \cap 5 \mathrm{~h}$ & $2.5 \mathrm{~h} \cup 5 \mathrm{~h}$ & $\begin{array}{l}\text { Regulation }{ }^{*} \text { and } \\
\text { observations }\end{array}$ \\
\hline Antibiotic resistance & Total & 56 & 3 & 2 & 2 & 3 & Down, lactamases \\
\hline \multirow{7}{*}{ Phages } & PPpW & 12 & 0 & 0 & 0 & 0 & No DEGs \\
\hline & phiCTX & 25 & 2 & 3 & 2 & 3 & Up \\
\hline & F10 & 62 & 1 & 9 & 0 & 10 & Up \\
\hline & JBD44 & 105 & 34 & 65 & 34 & 65 & Up \\
\hline & JDO24 & 59 & 4 & 7 & 4 & 7 & Up \\
\hline & phi3 & 45 & 0 & 0 & 0 & 0 & No DEGs \\
\hline & Total & 308 & 41 & 84 & 40 & 85 & - \\
\hline \multirow{12}{*}{ Virulence factors } & Adherence & 96 & 11 & 19 & 11 & 19 & Down \\
\hline & Antimicrobial activity & 17 & 1 & 6 & 1 & 6 & Up, phenazines \\
\hline & Antiphagocytosis & 25 & 0 & 0 & 0 & 0 & No DEGs \\
\hline & \begin{tabular}{|l|} 
Phospholipases \\
\end{tabular} & 3 & 0 & 0 & 0 & 0 & No DEGs \\
\hline & Biosurfactant & 3 & 0 & 0 & 0 & 0 & No DEGs \\
\hline & Iron uptake & 28 & 0 & 1 & 0 & 1 & Up, Pyochelin \\
\hline & Protease & 4 & 1 & 2 & 1 & 2 & Up, elastases \\
\hline & Quorum sensing & 5 & 0 & 1 & 0 & 1 & Up, RhlR \\
\hline & $\begin{array}{l}\begin{array}{l}\text { Regulation GacS/GacA } \\
\text { system }\end{array} \\
\end{array}$ & 2 & 0 & 0 & 0 & 0 & No DEGs \\
\hline & Secretion system & 63 & 0 & 2 & 0 & 2 & Down, T3SS \\
\hline & \begin{tabular}{|l|} 
Toxins \\
\end{tabular} & 4 & 0 & 1 & 0 & 1 & Up, hydrogen cyanide \\
\hline & Total & 250 & 13 & 32 & 13 & 32 & - \\
\hline
\end{tabular}

Table 2. Comparison of DEGs of PaeAG1 at 2.5 and $5 \mathrm{~h}$ after treatment with ciprofloxacin, and specific phages or categories of virulence factors, including shared genes (intersection) and total genes at both times (union), the regulation and the type of elements. ${ }^{*}$ Based in $\log \mathrm{FC}$ of genes for both times 2.5 and $5 \mathrm{~h}$. Type of elements is also shown.

In the case of the 250 known virulence factors of PaeAG1, 32 (12.8\%) were identified as DEGs at all of the assessed times (arrowheads of Fig. 4 and supplementary Figure S3). The virulence factors are mainly associated with adherence (19) and phenazines (6) genes (see Table 2). Regarding antibiotic resistance genes, only three out the 56 genes were found to be differentially expressed (Table 2).

A heatmap of normalized counts and gene clustering of the total 518 DEGs are shown in Fig. 3E. Well-defined clusters were found for genes and samples, showing similar expression patterns.

Out of all the DEGs at $2.5 \mathrm{~h}$, seven genes corresponded to transcription factors, including $p s r A, r p o H$ and $p r t N$. At $5 \mathrm{~h}, 14 \mathrm{DEGs}$ including $p s r A, r p o H, p r t N, r p o S, r h l R$ and $p \operatorname{tr} B$ were identified as transcription factors. All transcription factors activated at $2.5 \mathrm{~h}$ remained active at $5 \mathrm{~h}$ (Supplementary Table S2). Identification of regulators by a context-centric analysis revealed a total of 22 transcription factors modulating all the DEGs at $2.5 \mathrm{~h}$, and most of them are part of the 28 transcription factors recognized as DEGs at $5 \mathrm{~h}$ (see Supplementary Table S2).

Genes of the SOS response were not identified as DEGs. The $r p o S$ factor was up-regulated at $5 \mathrm{~h}$. Due to the preponderant role of LexA (SOS response) and RpoS as essential genes in the response to CIP in P. aeruginosa, we further investigated the DNA binding sites for these elements. The CollectTF database provided the consensus binding sequence for LexA as CTG-TATAA-ATATA-CAG, described by ${ }^{26}$. Analysis revealed the role of LexA modulating all 15 genes in the SOS response in P. aeruginosa, as well as other sequences at promoter regions of $p s r A$ (coding for a transcription factor as described before), grpE, hemO and other genes. In PaeAG1, psrA and $g r p E$ genes were up-regulated at 2.5 and $5 \mathrm{~h}$ after CIP treatment. For RpoS, no sequence information was available in CollectTF, therefore we used the RpoS-dependent promoter consensus sequence CTATACT found by $^{75}$. A total of 49 sites for RpoS were predicted to be associated with promoter regions of PaeAG1 genes, but none as DEGs in PaeAG1. 


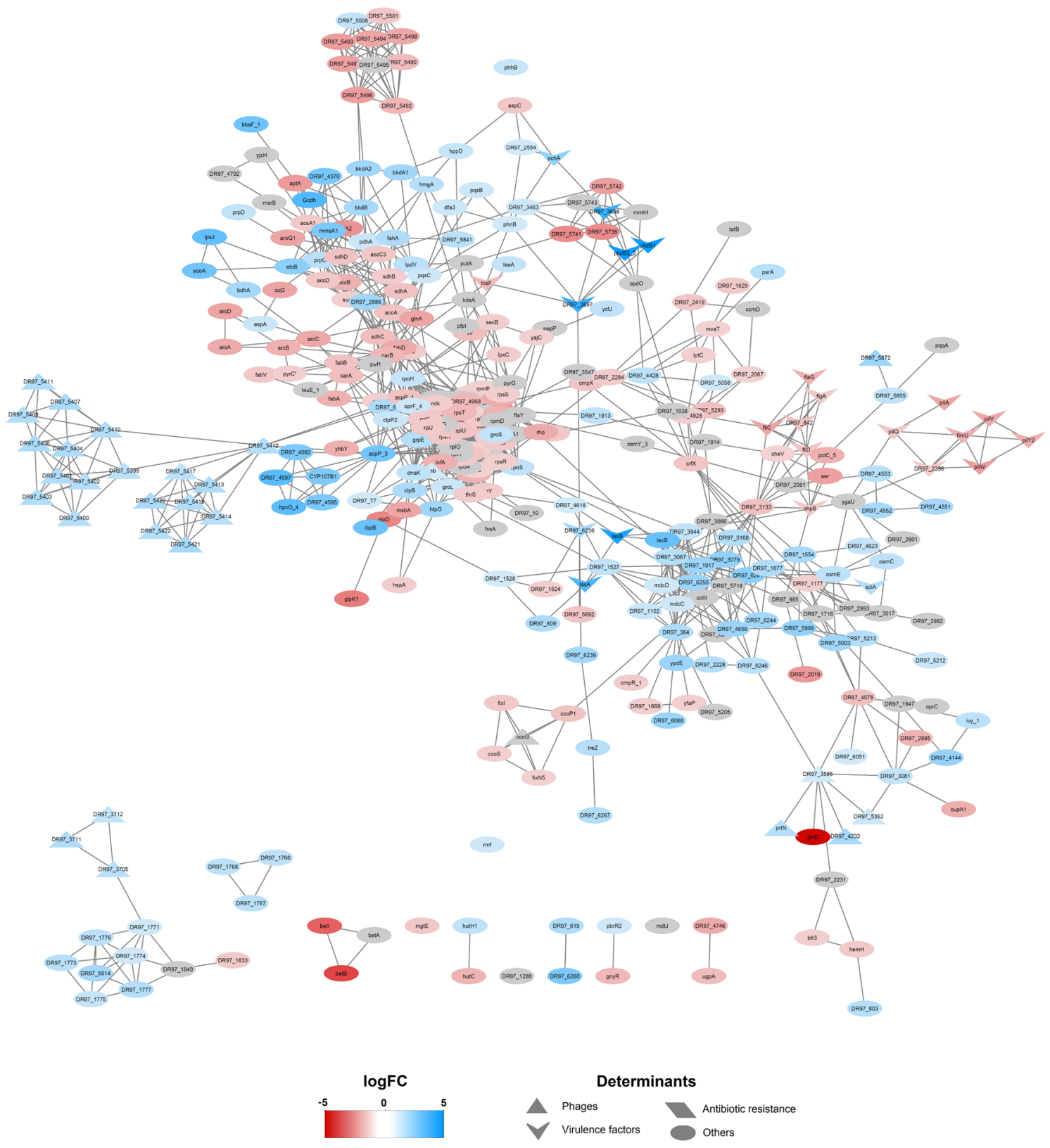

Figure 4. Gene-gene interaction (GGI) large scale network of differentially expressed genes in PaeAG1 after ciprofloxacin treatment, using a database-based method for prediction of interactions. Using STRINGdb, interactions between genes were predicted. To build the network all the DEGs in both times 2.5 and $5 \mathrm{~h}$ were included. A total of 342 genes resulted connected (66.0\% of all DEGs) with 1685 edges in total (not connected nodes are not shown). The $\log \mathrm{FC}$ is shown for $5 \mathrm{~h}$. Gray nodes represent genes that were differentially expressed only at time $2.5 \mathrm{~h}$ (i.e. no logFC value is displayed at time $5 \mathrm{~h}$ ). Details of the network by time is shown in supplementary Figure S3. Phages genes, virulence factors and antibiotic resistance genes are represented as triangles, arrowheads and rhomboids, respectively. Down-regulation (red tones) and up-regulation (blue tones).

Networks analysis shows pleiotropic effects of CIP exposure in PaeAG1. Using a top-down systems biology approach, a large scale GGI network of DEGs was built to identify molecular determinants associated with the response to CIP in PaeAG1.

GGI predictions by a database-based model: All of the 518 DEGs were incorporated as nodes and edges (high confidence connections or interactions). A total of 342 (66.0\% of all DEGs) nodes were found to be connected with at least one other gene, as well as 1685 edges were established (Fig. 4). When selecting DEGs for each time, 248 nodes $(69.9 \%)$ of the 355 DEGs at $2.5 \mathrm{~h}$ were connected with a total of 1,156 edges (supplementary Figure S2A). Out of all the 438 DEGs at 5 h, $284(64.8 \%)$ were connected with 1,041 edges in total (supplementary Figure S2B).

As shown in Fig. 4, some determinants of virulence factors (adherence) and antibiotic resistance genes showed a down-regulation after CIP treatment, meanwhile, phage genes and other virulence factors (phenazines) were 
A

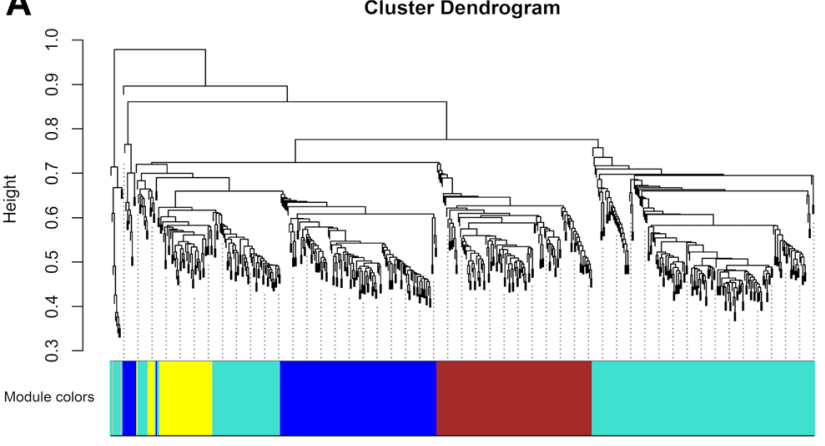

B

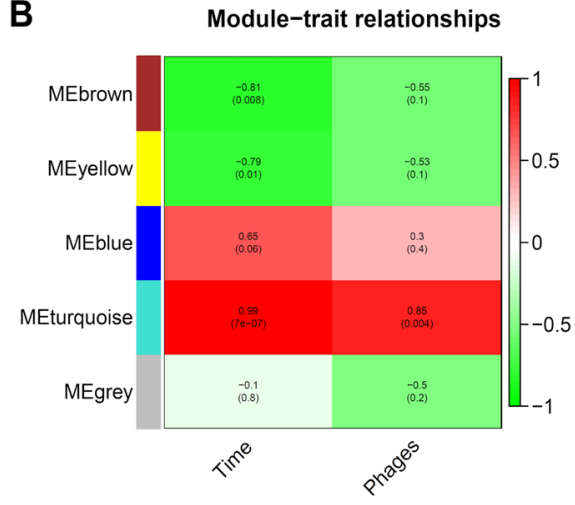

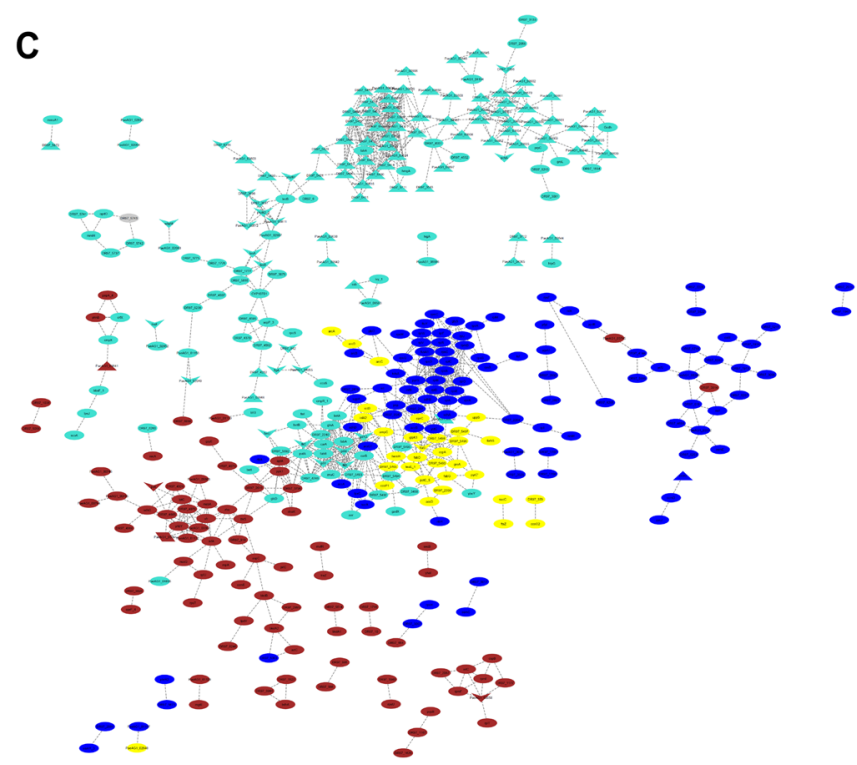

Figure 5. Co-expression analysis to identify modules of genes and the data-driven co-expression network in PaeAG1 after Ciprofloxacin treatment. (A) Modules identification (clusters by colors) using correlated expression genes (along times $0,2.5$ and $5 \mathrm{~h}$ ) and clustering analysis after WGCNA was implemented. (B) Association of modules to traits, showing relations between turquoise and blue modules with exposure time to antibiotic and phages induction. (C) Data-driven co-expression network using correlation of gene expression by WGCNA analysis (correlation $>98.5 \%$ ). A total of 388 DEGs were found to be connected, with a total of 1,073 edges. Only correlated genes are shown. More details in supplementary Figure S3A. Phages genes, virulence factors and antibiotic resistance genes are represented as triangles, arrowheads and rhomboids, respectively.

found to be up-regulated. In addition, gene clusters of highly connected DEGs showed the same expression pattern, suggesting a coordinated regulation.

The observed unconnected genes (107 DEGs for $2.5 \mathrm{~h}$ and 154 for $5 \mathrm{~h}$ ) are inherent to limitations in the database (incomplete inclusion of phage genes) or the current state of the gene annotation (without information, hypothetical protein, etc.). To improve the associations between genes creating more connections, a data-driven co-expression analysis was run.

Co-expression analysis. Modules of highly connected genes (represented using color groups) were created using normalized counts for all the 518 DEGs. As shown in Fig. 5A, genes were clustered into four main modules, showing similar expression along samples. The number of genes belonging to the turquoise module was 239, 124 for blue, 114 brown and 39 for yellow module. In the co-expression network (Fig. 5C), a total of 388 DEGs (74.9\% of the 518 DEGs) were found to be connected, with a total of 1,073 edges. Of these interactions, 385 were also found using the database-based model and 688 novel gene interactions were suggested by our coexpression analysis. The turquoise module includes most of the phage genes and virulence factors.

Integrated GGI network of DEGs. Integration of predicted connections between genes by both the databasebased model and co-expression analysis was done to build a definitive large scale network, shown in Fig. 6. A total of 449 (86.7\%) of DEGs were connected, in contrast with the 342 nodes from the preliminary network, an increment of $\sim 20 \%$. In addition, 2,373 edges were identified, 1685 from the database-based method (solid lines in the network) and the 688 new interactions suggested by the co-expression analysis (dashed lines). Further- 


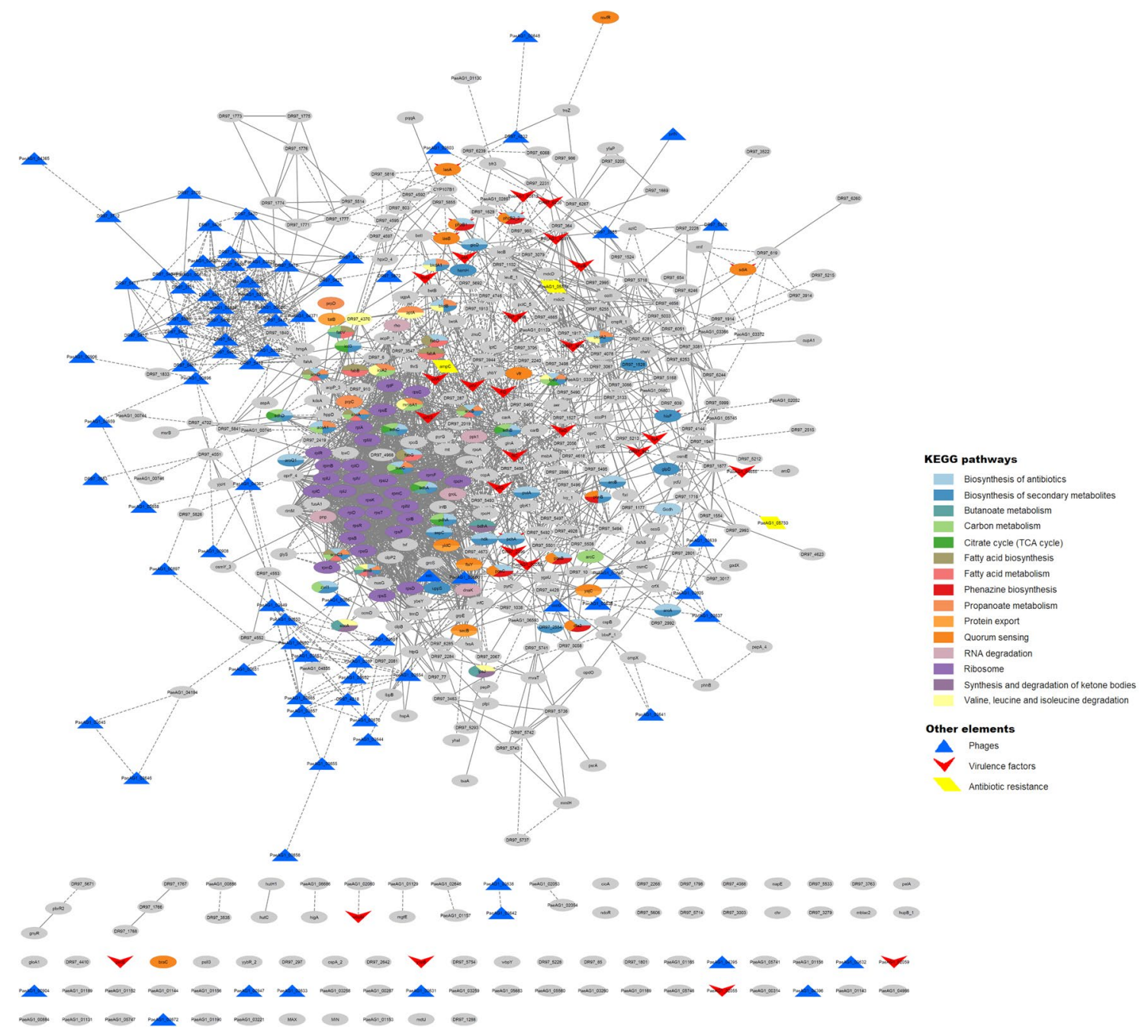

Figure 6. Definitive large scale network of DEGs, identification of hub genes and associated groups in PaeAG1 after treatment with ciprofloxacin. Network showing all 518 DEGs genes and their interactions (449 genes have at least one connection). Known interactions according to STRINGdb (database-based method) are shown as solid lines and data-driven interactions according to data-driven co-expression analysis as dashed lines. Enriched nodes associated to KEGG annotation are colored according to each pathway (more details in Table 3). Phages genes, virulence factors and antibiotic resistance genes are represented as triangles, arrowheads and rhomboids, respectively. Other genes are represented as ellipses.

more, a separated cluster was observed with high connectivity between phage genes (cluster of blue triangles, Fig. 6 left top). Remarkably, this cluster appears to have a critical bottleneck at the fahA gene, since many genes are connected to this node but, for the majority of the cluster nodes, this gene is the only connection to the rest of the network. Thus, the cluster becomes a clearly separated module. In addition, another smaller and less distinct cluster of phage genes was formed (Fig. 6 left down).

The same GGI network is presented in supplementary Figure S4A to show the distribution of genes by coexpression modules. A high functional interaction of genes across different clusters is observed. The logFC values at time $5 \mathrm{~h}$ are shown in the network in Figure S4B.

Enrichment analysis. In order to gain insight about the biological meaning of DEGs, gene set enrichment analysis (GSEA) was performed. The 518 DEGs were shown to be implemented in a total of 15 KEGG pathways (Figs. 6 and 7, and Table 3). The enriched pathways included ribosomal functions, RNA degradation, biosynthesis of antibiotics, fatty acids metabolism, propanoate metabolism, fatty acids biosynthesis, quorum sensing, amino acid degradation, carbon metabolism and citrate cycle, butanoate metabolism, phenazine biosynthesis, among others (see Fig. 7). Details of gene counts, FDR and regulation are shown in Table 3. Additionally, pathways by co-expression modules (Table 3 ) showed that some of them are enriched in specific pathways. For example, the blue module is down-regulated for ribosomal activity and RNA degradation (exclusive functions for this module), meanwhile the yellow module has multiple but tightly related pathways, most of them associated to interconnected metabolism pathways, down-regulated. 
A

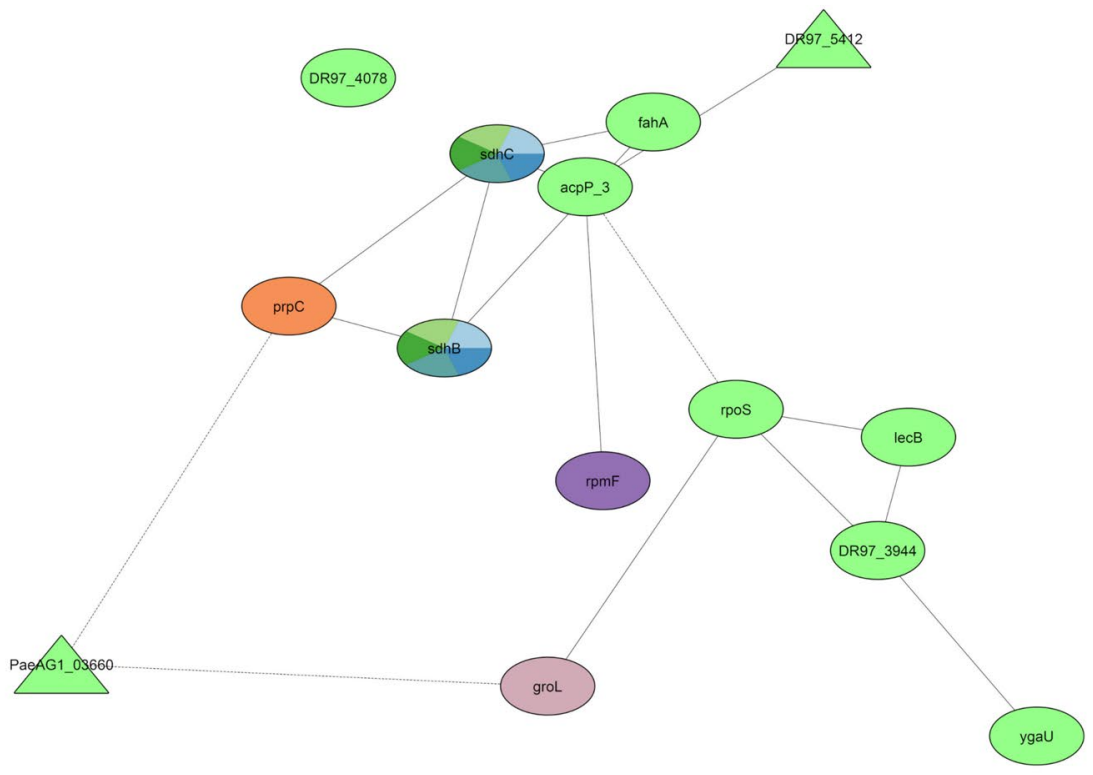

B

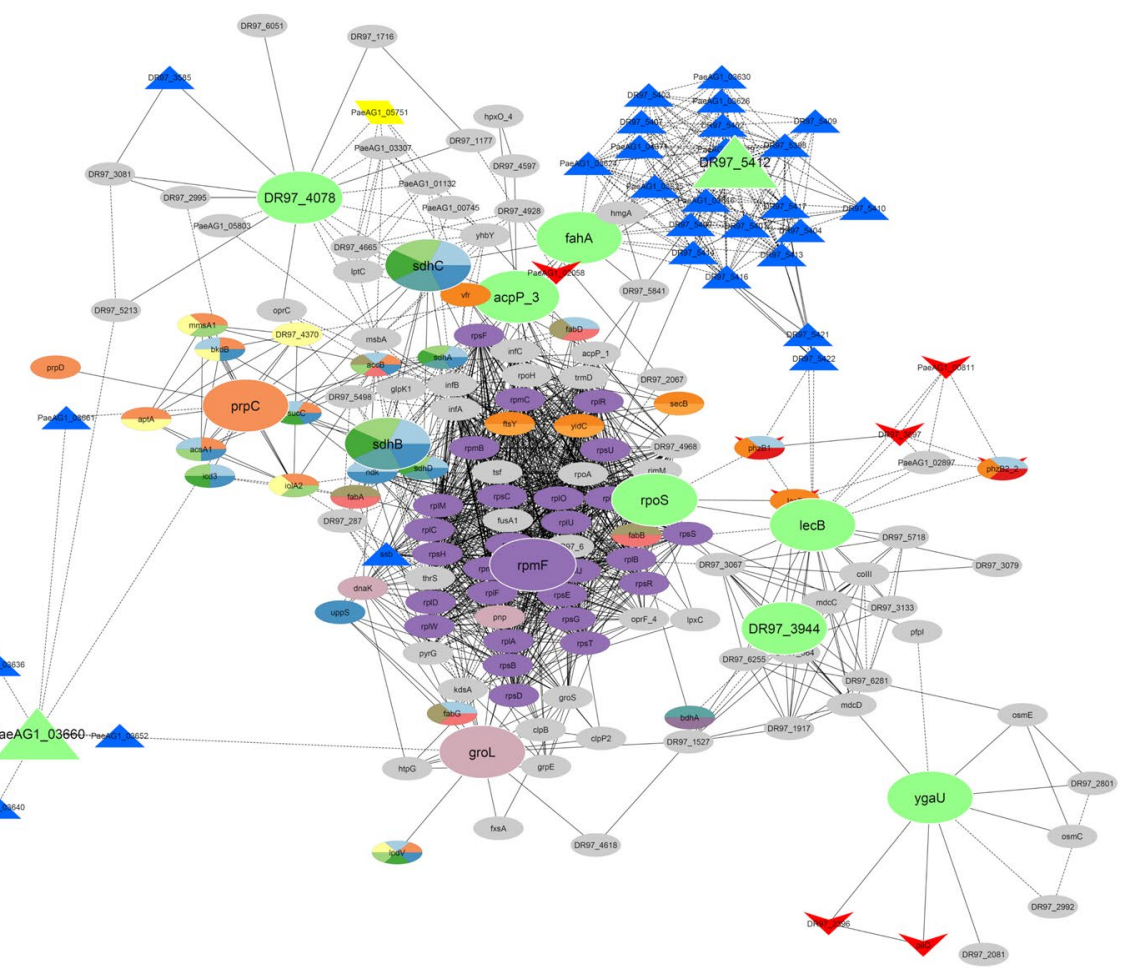

Figure 7. Identification of hub genes and first-stage subnetwork of their associated groups in PaeAG1 after treatment with ciprofloxacin. (A) Hub genes identification using cytoHubba (betweenness and bottleneck methods) in the network of DEGs (large nodes). Details in Table 4. (B) Subnetwork of nodes that directly interact with the 14 hub genes were used to build a first-stage elements network. Details of node shapes and colors are the same as described in Fig. 6.

Only 14 hub genes are able to represent the key pathways regulated by CIP in PaeAG1. With the aim of identifying an inter-modular key or central genes in the DEGs network of PaeAG1 after exposure to CIP, an analysis of hub gene identification was conducted. This approach revealed 14 connected hub genes (Fig. 7A and details in Table 4). Two genes, identified as PaeAG1_03660 and PaeAG1_03610, are part of the phage JBD44 and they were up regulated at $5 \mathrm{~h}$. Topologically, they are part of the two identified phage gene clusters in the main network (Fig. 6). Two genes, $s d h B$ and $s d h C$, (down-regulated) have functions related to 


\begin{tabular}{|c|c|c|c|c|c|c|c|c|}
\hline \multirow[b]{2}{*}{ KEGG term ID } & \multirow[b]{2}{*}{ Term description } & \multirow[b]{2}{*}{ Total gene count } & \multicolumn{2}{|l|}{ DEGs $2.5 \mathrm{~h}$} & \multicolumn{2}{|l|}{ DEGs $5 \mathrm{~h}$} & \multirow[b]{2}{*}{ Modules } & \multirow{2}{*}{$\begin{array}{l}\text { Regulation (\% } \\
\text { DEGs) }\end{array}$} \\
\hline & & & Observed gene count & FDR & Observed gene count & FDR & & \\
\hline paeb01130 & $\begin{array}{l}\text { Biosynthesis of } \\
\text { antibiotics }\end{array}$ & 266 & 30 & 0.0015 & 34 & 0.00047 & Brown, Yellow & Down $(61 \%)$ \\
\hline paeb01110 & $\begin{array}{l}\text { Biosynthesis of sec- } \\
\text { ondary metabolites }\end{array}$ & 320 & 30 & 0.0352 & 31 & 0.0205 & Yellow & Down $(70 \%)$ \\
\hline paeb00650 & Butanoate metabolism & 37 & 8 & 0.0133 & 9 & 0.0068 & Yellow & Down $(55 \%)$ \\
\hline paeb01200 & Carbon metabolism & 126 & 15 & 0.0258 & 18 & 0.0068 & Yellow & Down $(80 \%)$ \\
\hline paeb00020 & $\begin{array}{l}\text { Citrate cycle (TCA } \\
\text { cycle) }\end{array}$ & 30 & 7 & 0.0158 & 8 & 0.0068 & Yellow & Down $(75 \%)$ \\
\hline paeb00061 & Fatty acid biosynthesis & 27 & 7 & 0.0131 & 9 & 0.0014 & Yellow & Down $(100 \%)$ \\
\hline paeb01212 & Fatty acid metabolism & 49 & 8 & 0.0309 & 10 & 0.0068 & Yellow & Down (90\%) \\
\hline paeb00405 & $\begin{array}{l}\text { Phenazine biosyn- } \\
\text { thesis }\end{array}$ & 20 & 5 & 0.0309 & 6 & 0.0127 & Brown & Up $(100 \%)$ \\
\hline paeb00640 & $\begin{array}{l}\text { Propanoate metabo- } \\
\text { lism }\end{array}$ & 47 & 12 & 0.00061 & 16 & $3.87 \mathrm{e}-06$ & Brown, Yellow & Variable $(50 / 50)$ \\
\hline paeb03060 & Protein export & 15 & 5 & 0.026 & 3 & 0.0014 & Yellow & Down (100\%) \\
\hline paeb02024 & Quorum sensing & 86 & 11 & 0.0317 & 14 & 0.0068 & Brown & Up (69\%) \\
\hline paeb03010 & Ribosome & 55 & 27 & $1.95 \mathrm{e}-14$ & 27 & $2.63 e-13$ & Blue & Down (100\%) \\
\hline paeb03018 & RNA degradation & 17 & 5 & 0.0258 & 5 & 0.0273 & Blue & Down $(60 \%)$ \\
\hline paeb00072 & $\begin{array}{l}\text { Synthesis and deg- } \\
\text { radation of ketone } \\
\text { bodies }\end{array}$ & 10 & 4 & 0.0258 & 4 & 0.0273 & Brown, Turquoise & Up (100\%) \\
\hline paeb00280 & $\begin{array}{l}\text { Valine, leucine and } \\
\text { isoleucine degradation }\end{array}$ & 46 & 11 & 0.0015 & 11 & 0.0023 & Brown, Turquoise & Up (82\%) \\
\hline
\end{tabular}

Table 3. Pathways related to DEGs network of PaeAG1 exposed to ciprofloxacin, according to KEGG annotation. Annotation of modules of co-expressed genes and the general regulation are also included. ${ }^{\star}$ Based on $\log \mathrm{FC}$ of DEGs at both times 2.5 and $5 \mathrm{~h}$.

carbon and butanoate metabolism, and biosynthesis of secondary metabolites. Interestingly, the ribosomal protein L32 ( $r p m F$, down-regulated), a chaperonin ( $g r o L$, up-regulated) and the sigma factor ( $r p o S$, up-regulated) were also identified as single molecular determinants of the network. Also, the fahA gene, which was previously recognized as a bottleneck for the phage genes cluster and coding for fumarylacetoacetase enzyme, was identified as a hub gene.

Analysis of gene clusters of first-stage connected genes (Fig. 7B) showed not only the same profile of enriched pathways for those hub genes (Fig. 7A), but also other pathways such as lipids metabolism, phenazine biosynthesis, quorum sensing and others. These groups include many elements of phages, virulence factors and multiple uncharacterized genes, as well as one antibiotic resistance gene (PaeAG1_05751). The logFC values at time $5 \mathrm{~h}$ are shown in Figure S4C.

Six hub genes were consistently identified by both bottleneck and betweenness approaches (Table 4). Together with $r p o S$ and groL, eight hub genes (57\%) are part of the turquoise module, and all of them are up-regulated by CIP. All other genes are part of the brown (4) and blue modules (2). Only four genes were found to be down regulated, three of them belonging to the brown module.

To compare the expression profiles of hub genes to other studies, we included information in Table 4 of the effect of perturbations or stressors of $P$. aeruginosa in the modulation of gene expression. Similar effects of CIP on hub genes were found when comparing our results to a previous report ${ }^{26}$. The effects of azithromycin seem to be opposite to CIP for these genes. More variable results were found for other perturbations (e.g. colistin, copper and $\mathrm{H}_{2} \mathrm{O}_{2}$ ); and $l e c B$ was the only hub gene that was up-regulated for all perturbations.

Thus, as expected, hub genes are strongly linked to elements of highly connected gene clusters and at the same time with the key pathways in response to CIP. Together, these three elements (hub genes, gene clusters and enriched pathways) represent the determinants of the response to CIP in PaeAG1, many of them related to the bacterial growth modulation, as initially hypothesized.

Concentration dependent effect of CIP in PaeAG1 phage induction. According to transcriptomic analysis, phage genes were up-regulated under $12.5 \mu \mathrm{g} / \mathrm{mL}$ CIP treatment in PaeAG1. To validate these results at phenomic level, evaluation of lytic plaque formation was done using a phage plaque assay. As shown in Fig. 8A, after treatment with $12.5 \mu \mathrm{g} / \mathrm{mL}$ CIP, phage induction was increased by tenfold $(1,000 \mathrm{PFU} / \mathrm{mL})$ with respect to control condition without antibiotics, in concordance with the molecular findings. More drastic changes were evidenced for higher concentrations, where more than 10000 or $100000 \mathrm{PFU} / \mathrm{mL}$ were quantified for PaeAG1 after treatment with 25.0 and $50.0 \mu \mathrm{g} / \mathrm{mL}$ CIP concentrations, respectively. Figure $8 \mathrm{C}$ shows phage plaques on culture plate during in vitro assays. Unlike CIP, when the same analysis was done for imipenem and tobramycin (supplementary assay), no induction was evidenced. Indeed, a slight reduction was observed for imipenem (Supplementary Figure S1C-E, right). 


\begin{tabular}{|c|c|c|c|c|c|c|c|c|c|}
\hline $\begin{array}{l}\text { PaeAG1 Locus } \\
\text { ID }\end{array}$ & Gene name & $\begin{array}{l}\text { Betweenness } \\
\text { score }^{\star}\end{array}$ & \begin{tabular}{|l|}
$\begin{array}{l}\text { Bottleneck } \\
\text { score }\end{array}$ \\
\end{tabular} & $\operatorname{logFC} 2.5 h^{*}$ & $\operatorname{logFC} 5 h^{*}$ & $\begin{array}{l}\text { Co-expression } \\
\text { module }\end{array}$ & $\begin{array}{l}\text { KEGG } \\
\text { Annotation }^{* *}\end{array}$ & $\begin{array}{l}\text { Annotation } \\
\text { details }\end{array}$ & $\begin{array}{l}\text { Other } \\
\text { studies*** }\end{array}$ \\
\hline PaeAG1_01864 & acpP (PA2966) & $6,268.3$ & 17 & 2.64 & 3.63 & Turquoise & $\begin{array}{l}\text { Metabolic } \\
\text { pathways, } \\
\text { biosynthesis of } \\
\text { antibiotics }\end{array}$ & $\begin{array}{l}\text { Acyl carrier pro- } \\
\text { tein; fatty acid } \\
\text { biosynthesis }\end{array}$ & $\begin{array}{l}\uparrow \mathrm{AZM}, \\
\uparrow \mathrm{CIP} \text { COL }\end{array}$ \\
\hline PaeAG1_06246 & ygaU & $6,340.4$ & - & 1.79 & 0.9 & Blue & - & $\begin{array}{l}\text { LysM domain/ } \\
\text { BON superfam- } \\
\text { ily protein }\end{array}$ & - \\
\hline PaeAG1_04068 & sdhB (PA1584) & $6,401.4$ & - & -1.37 & -1.17 & Blue & \begin{tabular}{|l|} 
Biosynthesis \\
of antibiotics, \\
Carbon metabo- \\
lism, Citrate \\
cycle (TCA \\
cycle), Butanoate \\
metabolism, \\
Biosynthesis \\
of secondary \\
metabolites
\end{tabular} & $\begin{array}{l}\text { Succinate dehy- } \\
\text { drogenase and } \\
\text { fumarate reduc- } \\
\text { tase iron-sulfur } \\
\text { family protein }\end{array}$ & $\begin{array}{l}\uparrow \mathrm{COL} \text { AZM } \\
\downarrow \mathrm{CIP} \mathrm{Cu}\end{array}$ \\
\hline PaeAG1_04991 & prpC (PA0795) & $6,485.7$ & 14 & 1.58 & 1.7 & Turquoise & $\begin{array}{l}\text { Propanoate } \\
\text { metabolism }\end{array}$ & $\begin{array}{l}\text { Belongs to the } \\
\text { citrate synthase } \\
\text { family }\end{array}$ & $\begin{array}{l}\uparrow \mathrm{H}_{2} \mathrm{O}_{2} \mathrm{CIP} \downarrow \\
\mathrm{AZM} \uparrow \mathrm{COL}\end{array}$ \\
\hline PaeAG1_03610 & DR97_5412 & $7,285.4$ & 15 & 0.9 & 1.84 & Turquoise & - & $\begin{array}{l}\text { Phage: JBD44; } \\
\text { Tail tape meas- } \\
\text { ure protein }\end{array}$ & - \\
\hline PaeAG1_05221 & $\begin{array}{l}\text { groL or groEL } \\
\text { (PA4385) }\end{array}$ & $8,440.2$ & - & 1.16 & 1.21 & Turquoise & $\begin{array}{l}\text { RNA degrada- } \\
\text { tion }\end{array}$ & \begin{tabular}{l|}
$60 \mathrm{kDa}$ chaper- \\
onin; Prevents \\
misfolding and \\
promotes the \\
refolding and \\
proper assembly \\
of unfolded \\
polypeptides \\
generated under \\
stress conditions
\end{tabular} & $\begin{array}{l}\uparrow \mathrm{CIP} \mathrm{Cu} \\
\downarrow \mathrm{AZM} \uparrow \mathrm{H}_{2} \mathrm{O}_{2}\end{array}$ \\
\hline PaeAG1_04071 & sdhC (PA1581) & $8,716.8$ & - & -1.68 & -1.54 & Brown & \begin{tabular}{|l|} 
Biosynthesis \\
of antibiotics, \\
Carbon metabo- \\
lism, Citrate \\
cycle (TCA \\
cycle), Butanoate \\
metabolism, \\
Biosynthesis \\
of secondary \\
metabolites
\end{tabular} & \begin{tabular}{|l|} 
Succinate \\
dehydrogenase, \\
cytochrome b556 \\
subunit
\end{tabular} & $\begin{array}{l}\uparrow \mathrm{AZM} \\
\downarrow \mathrm{CIP} \mathrm{Cu}\end{array}$ \\
\hline PaeAG1_03660 & PaeAG1_03660 & $9,477.2$ & 17 & 1.05 & 1.23 & Turquoise & - & Phage: JBD44 & - \\
\hline PaeAG1_03555 & fahA (PA2008) & $11,245.9$ & 16 & 1.19 & 1.93 & Turquoise & \begin{tabular}{|l|}
$\begin{array}{l}\text { Tyrosine } \\
\text { metabolism }\end{array}$ \\
\end{tabular} & $\begin{array}{l}\text { Fumarylacetoac- } \\
\text { etase }\end{array}$ & $\begin{array}{l}\uparrow \mathrm{CIP} \uparrow \mathrm{COL} \\
\downarrow \mathrm{Cu} \mathrm{AZM}\end{array}$ \\
\hline PaeAG1_01837 & lecB (PA3361) & $13,150.8$ & 17 & 1.71 & 3.88 & Turquoise & Quorum sensing & $\begin{array}{l}\text { fucose-binding } \\
\text { lectin PA-IIL }\end{array}$ & $\uparrow$ CIP COL AZM \\
\hline PaeAG1_01229 & DR97_3944 & - & 15 & 1.3 & 1.45 & Brown & - & $\begin{array}{l}\text { Uncharacterized } \\
\text { protein }\end{array}$ & - \\
\hline PaeAG1_01591 & $\begin{array}{l}\text { rpoS } \\
\text { (PA3622) }\end{array}$ & - & 15 & 1.03 & 1.49 & Turquoise & $\begin{array}{l}\text { Transcription } \\
\text { machinery }\end{array}$ & $\begin{array}{l}\text { RNA polymerase } \\
\text { sigma factor } \\
\text { RpoS }\end{array}$ & $\begin{array}{l}\uparrow \mathrm{COL} C \mathrm{CIP} \\
\downarrow \mathrm{AZM} \uparrow \mathrm{Cu}\end{array}$ \\
\hline PaeAG1_01361 & DR97_4078 & - & 19 & -1.22 & -1.48 & Brown & - & $\begin{array}{l}\text { Uncharacterized } \\
\text { protein }\end{array}$ & - \\
\hline PaeAG1_02250 & rpmF (PA2970) & - & 22 & -1.17 & -1.39 & Brown & Ribosome & \begin{tabular}{|l|} 
Ribosomal pro- \\
tein L32; Belongs \\
to the bacterial \\
ribosomal \\
protein bL32 \\
family
\end{tabular} & $\begin{array}{l}\downarrow \mathrm{CIP} \mathrm{H}_{2} \mathrm{O}_{2} \\
\downarrow \mathrm{COL} \mathrm{Cu}\end{array}$ \\
\hline
\end{tabular}

Table 4. Characterization of hub genes in the DEGs network of PaeAG1 after treatment with ciplofloxacin. ${ }^{\star}$ Cases with gray numbers refer to genes which were no selected as a DEG at that time (logFC and adjusted $p$-value). ${ }^{* *}$ Cases with “-” refer to no annotation information. ${ }^{* * *}$ Results from other studies: $\uparrow$ up-regulated, $\downarrow$ down-regulated, $\uparrow$ variable regulation or “- " no information. All results from GEO-NCBI according to stress conditions: $\mathrm{Cu}$ (copper) from (Teitzel et al., 2006), CIP (ciprofloxacin) from (Cirz, O’Neill, Hammond, Head, \& Romesberg, 2006), COL (colistin) from (Cummins, Reen, Baysse, Mooij, \& O'Gara, 2009), AZM (Azithromycin) from (Kai et al., 2009) and $\mathrm{H}_{2} \mathrm{O}_{2}$ (hydrogen peroxide) from (Chang, Small, Toghrol, \& Bentley, 2005). 
A

Phage plaques

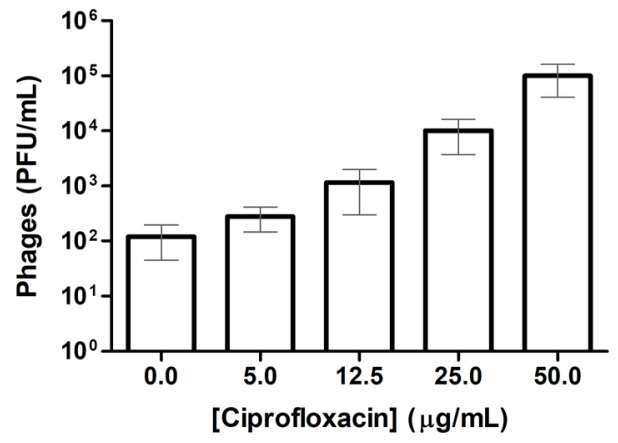

B

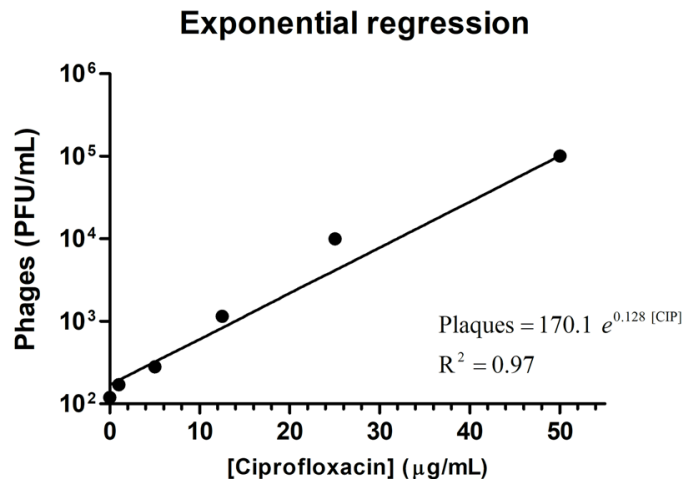

C

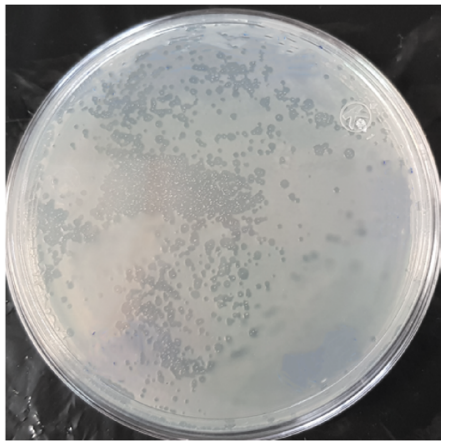

Figure 8. Phage plaques assay of PaeAG1 after exposure to ciprofloxacin. (A) Phages of PaeAG1 are induced under CIP exposure, with a pattern of higher induction of phage plaques at higher concentration of the drug, evidenced with an exponential regression as shown in (B). (C) Example of visualization of phage plaques on culture plate during in vitro assays.

Analysis of module genes to traits of PaeAG1 (phage production and time after CIP exposure) is presented in Fig. 5B. This analysis revealed a significant association of gene expression of the blue module, with changes at $2.5 \mathrm{~h}$ after CIP treatment and the low phage induction at this same time point. In a similar way, the turquoise module was significantly associated with changes of gene expression at $5 \mathrm{~h}$ and stronger phage induction. Other modules were not directly associated with these traits.

Altogether, these results indicate that phage induction in PaeAG1 is strongly dependent on CIP concentration, as shown with an exponential regression $\left(\mathrm{R}^{2}=0.97\right)$ in Fig. $8 \mathrm{~B}$.

\section{Discussion}

P. aeruginosa is a remarkable organism that can successfully resist, adapt, and survive in a wide variety of environments $^{29}$. This versatility is conferred by the large proportion $(>8 \%)$ of regulatory genes encoded in its large genome $\left(6-7.5 \mathrm{Mb}, 7.2 \mathrm{Mb}\right.$ in the case of PaeAG1) ${ }^{5,22}$. This particular case of PaeAG1 strain is a high-risk ST-111 strain isolated from an immune-compromised patient in a Costa Rican Hospital, with resistance to multiple antibiotics including CIP and carbapenems. Although many P. aeruginosa strains are resistant to CIP ${ }^{6,10,12,48}$ and other antibiotics, the effects of sub-lethal concentrations on the development of antibiotic resistance had been ignored for decades due to the assumption that resistance emerges only with lethal concentrations $(>\mathrm{MIC})^{14}$.

Therefore, we evaluated the effect of different CIP concentrations on PaeAG1 growth rate (Fig. 2). We detected a concentration-dependent reduction of growth rate as the CIP concentration was increased, similar to another study with CIP in P. aeruginosa $a^{12}$. We then employed RNA-Seq analysis to investigate the influence of a subinhibitory CIP concentration on the gene expression of PaeAG1 and its relationship with the bacterial growth, similar to recent studies in P. aeruginos $a^{76,77}$ and other bacteria ${ }^{16,44,78-81}$. Differential expression analysis (Fig. 3) highlighted 518 DEGs at 2.5 and $5 \mathrm{~h}$. Contrasting results have been previously reported in P. aeruginosa after CIP exposure, with some variations attributed mainly to differences in CIP concentration, time after exposure and/or the technical approach ${ }^{12,26,48}$.

We used a top-down systems biology approach to build the interaction network across the 518 DEGs. Interactions were modeled using a database-based method and co-expression analysis. A total of 14 hub genes, gene clusters and 15 KEGG pathways were associated with the molecular response to CIP, many of them related to bacterial growth, in line with other studies ${ }^{26,82,83}$. Discovery and description of these strong relationships between genes provided not only biological insights of the molecular regulation under stress conditions ${ }^{42}$, but also helped to reduce data complexity to only several central elements ${ }^{40}$, as other studies in P. aeruginosa PAO ${ }^{47}$ and E. coli ${ }^{40}$. 
Sigma factor RpoS as a hub gene. Not surprisingly, one of the identified hub genes in PaeAG1 after CIP treatment was rpoS. This gene was only up-regulated at $5 \mathrm{~h}$ after exposure, suggesting a late regulation in comparison with other DEGs. RpoS is considered a master regulator of the general stress response ${ }^{35}$ which is induced when bacterial growth decreases, or under starvation, antibiotics and osmotic or oxidative stress ${ }^{18}$. In addition, RpoS participates in the protection of cellular macromolecules ${ }^{18}$, modulation of metabolism, virulence, and changes in cell envelope and morphology ${ }^{11}$. The overexpression of RpoS suggests that bacteria enter a stationary phase-like state upon stress conditions, as reported previously ${ }^{44}$. This is further supported by the observed significant lack of growth of bacteria under CIP treatment of various concentrations.

According to growth curves, PaeAG1 was in exponential phase at the time points used for the transcriptomic analysis (Fig. 2 and supplementary Figure S1A). This is a key point to ensure that RpoS induction (and all the response) is explained by the antibiotic and not due to stationary-phase entry (i.e. experimental design). The reliance of the observed changes on CIP treatment was further supported by the fact the curves at same conditions showed no changes for imipenem or tobramycin antibiotics (supplementary Figure S1C-E). Other fluoroquinolones were not tested for their effect on the production of phages in PaeAG1.

In addition, DNA binding site analysis using consensus sequence described in ${ }^{75}$ revealed 49 sites for RpoS in PaeAG1 genes, however none of these were found to be DEGs. In the same work, RpoS was regulating 772 genes at the stationary phase, of which 41 genes $(5 \%)$ were identified as DEGs in our study. Since our analysis was performed at the exponential phase, the small number of common genes could be attributed to growth phase differences in each study. In another study using a de novo approach to identify binding sites using ChIP-Seq, RpoS showed to have 199 binding motifs in P. aeruginosa PA14 ${ }^{37}$, including six transcription factors. In PaeAG1, 23 of these 199 genes corresponded to promoter regions of DEGs, including the RhlR and RpoS (itself) transcription factor genes. This suggests that $12 \%$ of the RpoS regulon was modulated by CIP in PaeAG1. Interestingly, context-centric analysis revealed that up to 28 transcription factors (including RpoS) are associated with the response to CIP, regulating gene expression with pleiotropic consequences and defining a crosstalk among factors in P. aeruginosa ${ }^{37}$.

On the other hand, the RpoS response contributes to the robustness of bacterial cells facing stress conditions, acting synergistically with the SOS response ${ }^{18}$. Although SOS response is known to be induced by CIP in P. aeruginos $a$ and other bacteria ${ }^{20,26,27,84}$, in this study the SOS response was not significantly induced in response to CIP treatment at 2.5 and $5 \mathrm{~h}$. The absence of SOS induction may be due to the timing and concentration of CIP treatment. In E. coli, dynamic models have shown that the time of response to cell stress is very fast, and stability of the SOS response can be achieved in minutes, around $30 \mathrm{~min}$ according to ${ }^{85}$ or up to 90 min according to ${ }^{86}$, until homeostasis is recovered or stronger stress responses are induced. Also, the SOS regulon of $P$. aeruginosa was established using a supra-inhibitory CIP concentration $(8 \times \mathrm{MIC})$ at times 30 and $120 \mathrm{~min}^{26}$. These differences in concentration and time $(0.4 \times \mathrm{MIC}$ at 2.5 and $5 \mathrm{~h}$ for PaeAG1) could explain absence of SOS elements as DEGs. Our results are similar to another proteomic study using P. aeruginosa; profiles at 1.5, 5.5 and $14.5 \mathrm{~h}$ after CIP treatment were evaluated, and neither LexA nor other SOS proteins were differentially expressed, except for RecA, which was found to be up-regulated ${ }^{87}$.

Phage induction as a response determinant. Regarding phage genes, two gene clusters with hub genes were defined in PaeAG1 after CIP treatment. Phage induction is known to be modulated upon stress conditions, including the SOS response ${ }^{88}$. As found recently for some antimicrobials, phage activity is product of pleiotropic regulation ${ }^{89}$. In the presence of sub-lethal concentrations of certain antibiotics, phages have been observed to be induced or to form larger phage plaques ${ }^{88,90}$. Under fluoroquinolones exposure, P. aeruginosa DNA is affected and the SOS response is triggered. In a similar manner to LexA, repressor cleavage reaction is stimulated by activated RecA, allowing virus assembly ${ }^{91,92}$, and killing of the bacterium ${ }^{93}$. In some cases, alternative RecAindependent mechanisms have been described ${ }^{91,94}$.

PaeAG1 has six prophages in the genome, including two complete elements ${ }^{5}$. After CIP exposure 85 phage genes were up-regulated, most of them from JBD44 ( 65 genes out of 105 JBD44 genes). In the co-expression analysis, when association between modules and traits was assessed, the turquoise module (Fig. 5) was significantly related to CIP exposure time and phage induction, indicating a coordinated gene expression activity belonging to this cluster/traits (Fig. 5B).

Although general information on PaeAG1 phages is scarce, there is evidence to suggest that JBD44 is one of the most prevalent in $P$. aeruginos $a^{95}$. Effects of JBD44 induction on growth have been previously described in P. aeruginosa PAO1, showing that JBD44 expression significantly decreased the growth of PAO1, unlike other phages ${ }^{96}$. Similarly, SOS-mediated phage induction has been reported in P. aeruginosa PAO $1^{12,26}$ and LESB58 ${ }^{97}$. In addition, effect evaluation of several antibiotics found that CIP and norfloxacin (another fluoroquinolone) caused a high level of phage induction, but variable results were found for other antibiotics ${ }^{92}$. As observed in our experiments, no induction was found for imipenem nor tobramycin (supplementary Figure S1C-E).

The underlying relationship between the up-regulation of multiple phage genes in PaeAG1 after CIP exposure and the effect on bacterial lysis was validated through the effect of CIP concentrations in the phage induction. A concentration-dependent effect of CIP on both growth curves (rate reduction, Fig. 2) and phage plaques formation (exponential increment, Fig. 8) was demonstrated. This validated the transcriptomic findings of upregulation of phage genes in PaeAG1.

In congruence with this and the enriched pathways in PaeAG1, it has been reported that cells can adapt to stresses by disrupting their own metabolism in such a way that will impair the success of phage activity ${ }^{98}$. This implies that effects are observed not only on the host cell fate but also modulation of different responses, including RpoS regulation. These changes can be a product of tight modulation of functions reliant on molecular interactions from both phage and bacteria ${ }^{99}$. Similarly, as phages generally appear to consume amino acid 
metabolites ${ }^{100}$, the bacterial up-regulation response of genes involved in amino acid catabolism has been suggested as a strategy for reducing the infection success ${ }^{98}$ and disrupting phage propagation ${ }^{100}$. Blasdel et al. 2017 found that $m a i A$, fahA, $h m g A$ and $h p d$ genes of tyrosine catabolism were up-regulated by $P$. aeruginosa during phage activity ${ }^{98}$. In our study, all four genes were up-regulated, including $f a h A$ as a hub gene and a bottleneck element for the main phage gene cluster, indicating a catabolic effect after exposure to CIP that may be related to phage induction. More details of the $f a h A$ gene are discussed later.

Although different possibilities of the regulation of phage genes have been suggested, in the case of PaeAG1 phages, most of the predicted phage genes cannot be associated with a putative function, as in other studies ${ }^{26}$. This complicates the interpretation of the results for particular genes ${ }^{99}$. Validation of phage induction at phenomic level in congruence with transcriptomic results suggests that modulation of phages by CIP (but not for imipenem or tobramycin as discussed before) in PaeAG1 is possible. This is particularly relevant since this strain is a ST-111 high-risk clone and a critical organism Priority 1 (resistant to carbapenems) according to $\mathrm{WHO}^{8}$. Modulation could be achieve targeting phage production as a therapeutic option, with the advantage that the induced phages are resident elements of the genome and not exogenous elements as in other studies. Thus, treatment of antibiotic-resistant bacterial infections can potentially be improved by using phage therapy and traditional antibiotics, regardless if cells are growing in biofilms or as planktonic bacteria ${ }^{88}$. In addition, phage therapy can be used as a bactericidal element against multiresistant strains ${ }^{93}$. However, this does not necessarily apply to all $P$. aeruginosa strains since phage induction in other cases (with different strains and antibiotics) have been shown to be variable ${ }^{92}$.

Other transcriptomic determinants. Of the 15 pathways recognized as enriched in PaeAG1 after CIP treatment, ribosomal activity, RNA degradation and several metabolic routes were prominently enriched with respect to others. Reduction in the abundance of ribosomal proteins and protein implicated in cell division over time indicate a shift by tolerant cells away from growth ${ }^{87}$, as it was evidenced by the changes in the growth curves under different CIP concentrations in PaeAG1. In the case of ribosomal activity, a cluster is clearly recognized in the whole network and the subnetwork of hub genes, where the $r p m F$ gene is the up-regulated hub element. The $r p m F$ gene encodes for the $50 \mathrm{~S}$ ribosomal subunit protein L32, which is responsible for protein synthesis and membrane lipid synthesis ${ }^{101}$. It is also involved in multidrug tolerance by modulating biofilm formation and persister cell induction ${ }^{102}$.

Regarding metabolism, several reports have shown a down-regulation of energy production and carbohydrates, amino acids and lipids metabolism ${ }^{15,36,87,103,104}$. Five hub genes ( $s d h B, s d h C, \operatorname{prp} C, \operatorname{acp} P$ and $\left.f a h A\right)$ are particularly associated with metabolism. For instance, $f a h A$ is key in the inhibition of amino acid metabolism ${ }^{105}$, coding for a fumarylacetoacetase necessary for the tyrosine catabolism pathway. In addition, fahA is a topological bottleneck in the networks (Fig. 6A-C), separating the main phage genes cluster from the rest of the nodes. As detailed before, regulation of this gene could be used to restrict amino acids access to the phage and thus restraining the full phage activity ${ }^{98}$.

In the case of RNA degradation pathways, we identified groL (or groEL) as a hub gene, a homolog of heat shock protein $60^{106}$. DnaK and GroL are major ubiquitous chaperones that play crucial roles in promoting protein folding during normal growth and under stress conditions ${ }^{107}$ such as oxidative stress, antibiotics or heat ${ }^{26,107,108}$. In PaeAG1, both chaperones were up-regulated.

In relation to virulence factors, CIP modulated adherence and phenazines. A total of 19 DEGs implicated in adherence were identified with down-regulation observed for LPS O-antigen, flagella, and type IV pili biosynthesis elements. Similar results were found for P. aeruginosa after CIP treatment in another study ${ }^{26}$. Under other stress conditions, this down-regulation has been suggested to be a mechanism to avoid biofilm formation as a possible way to escape as planktonic cells ${ }^{46}$ and, in general, to modulate mechanisms for colonization, survival and invasion within the host tissues ${ }^{93}$.

Regarding phenazines, six genes were up-regulated. This profile is associated with tolerance to oxidative stress, iron availability, biofilms, virulence and killing microbial competitors ${ }^{109}$. Phenazine biosynthesis is regulated by the $\mathrm{Rhl}^{76}$ and $\mathrm{PQS}^{110}$ quorum sensing systems in P. aeruginosa. The rhlR gene was found to be up-regulated, suggesting a possible regulation of the phenazines.

More details of specific genes and their relationship with other virulence factors, antibiotic resistance and other responses (all with few number of DEGs) are discussed in the supplementary material "Extended discussion: Other transcriptomic determinants of PaeAG1 in response to CIP".

Altogether, the transcriptomic analysis in PaeAG1 allowed us to identify key molecular determinants of the response to CIP, many of them related to the bacterial grown, such as RpoS and phage induction. This agrees completely with our hypothesis in which transcriptomic response to CIP was related to bacterial growth modulation. After a DNA damage response is induced by sub-inhibitory CIP treatment, there is a subsequent pathway modulation and transcriptional changes that define changes in the bacterial growth. A conceptual representation of these results is shown in Fig. 9, aiming to integrate our results, literature reports and possible unknown connections.

All these features are particularly relevant for high-risk strains, such as PaeAG1. As it has been suggested, the biological markers of $P$. aeruginosa high-risk clones could be useful for the future design of specific treatments and infection control strategies ${ }^{7}$. Thus, more detailed analyses are needed to study the different levels of transcriptomic regulation in PaeAG1, including targeted expression analysis, other stress conditions, genetic and phenotypic variability, validation of the effect and power of hub genes, explorations of the relationship between presence of specific virulence traits and severity, and phage induction as a potential therapy. 


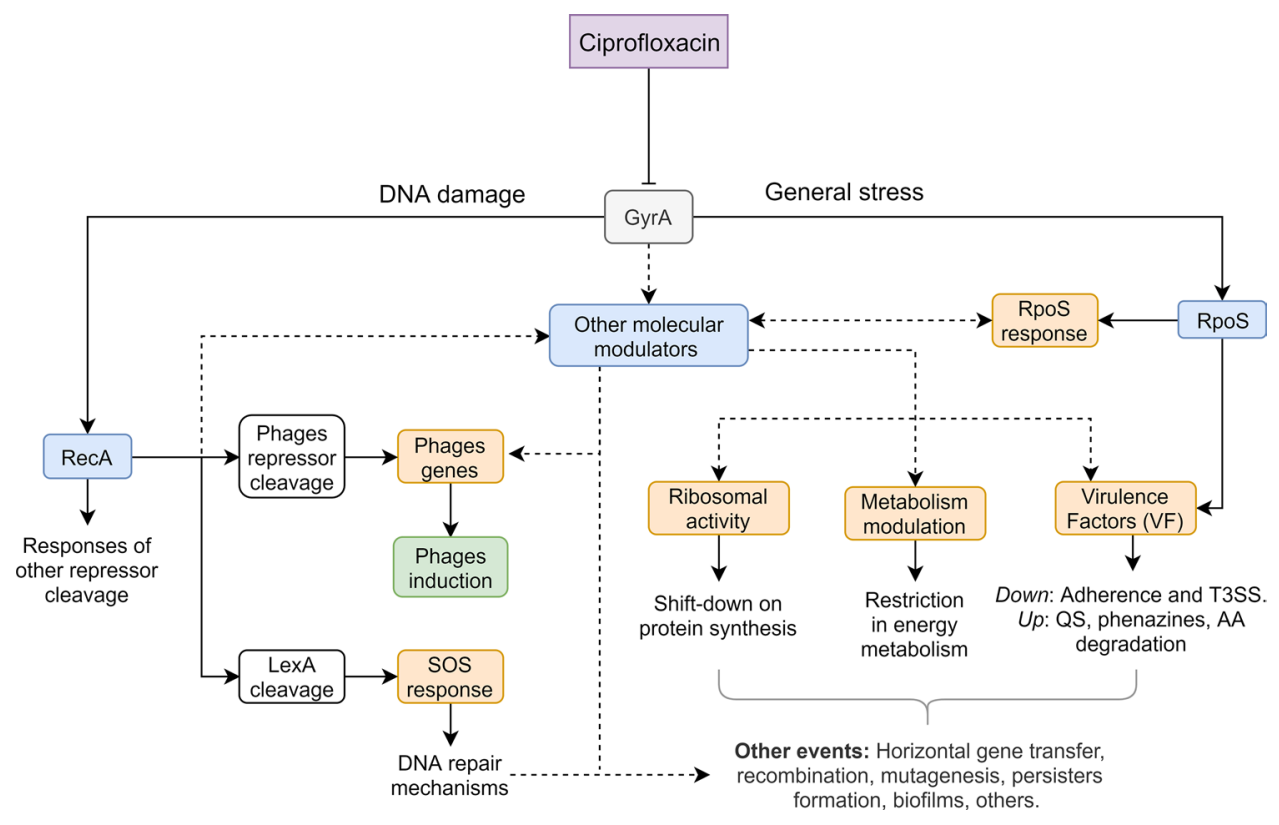

Figure 9. Conceptualization of effects of ciprofloxacin treatment in PaeAG1 at the molecular level. Effects of DNA damage triggers RecA increment, which cleaves different repressors such as LexA, inducing the SOS response, but also phages induction repressors, and other elements. The general stress induces the RpoS response, modulating different responses and virulence factors. Other modulators induce changes in the metabolic state of cells, expression of virulence factors, as well as the down-shift in ribosomal activity. Together, all changes imply modulation of multiple responses with pleiotropic effects at a molecular level and regulation of phenotypes to face the stress given by the antibiotic.

\section{Conclusions}

In this work, we report a concentration-dependent reduction of PaeAG1 growth rate upon increasing subinhibitory CIP concentrations by comparing growth curves. The RNA-Seq analysis of PaeAG1 after treatment with a sub-inhibitory CIP concentration allowed us to identify 518 DEGs along time at 2.5 and 5 h. Using a top-down systems biology approach, we identified diverse transcriptomic determinants: 14 hub genes, multiple gene clusters and 15 enriched pathways. These included down-regulation of pathways related to metabolism, ribosomal activity and adherence factors, most of them related to bacterial growth reduction. Phages, phenazines and specific virulence factors were found to be up-regulated. In most cases, hub genes and complex relationships were identified, showing pleiotropic effects that are mainly illustrated by clusters of highly connected genes. Two particular clusters of phages genes were up-regulated by CIP. Validation of CIP effects on phage induction was done at phenomic level with a phage plaque assay, showing an exponential induction as CIP was increased. To our knowledge, this is the first report of the analysis of CIP response in a ST-111 high-risk P. aeruginosa strain, in particular by a combined strategy using a top-down systems biology approach. This led us to identify transcriptomic determinants in response to CIP, including resident phages induction as a potential therapeutic strategy to overcome antibiotic resistance.

\section{Data availability}

The RNA-seq raw data and processed files of transcripts quantification are available at the NCBI Gene Expression Omnibus (GEO) database under accession number GSE139866. Processed data and scripts for bioinformatics analyses (RNA-Seq data, differential expression using DESeq2 and co-expression analyses) are available at https://github.com/josemolina6/PaeAG1_CIP_RNA-Seq). Genome sequence and annotation files in all required formats for mapping and quality control of the RNA-Seq reads alignment are available from our previous work at https://github.com/josemolina6/PaeAG1_genome. More details of the genome assembly and annotation in ${ }^{5}$.

Received: 11 March 2020; Accepted: 25 June 2020

Published online: 13 August 2020

\section{References}

1. Lyczak, J. B., Cannon, C. L. \& Pier, G. B. Establishment of Pseudomonas aeruginosa infection: Lessons from a versatile opportunist $1^{\star}$ Address for correspondence: Channing Laboratory, 181 Longwood Avenue, Boston, MA 02115, USA. Microbes Infect. 2, 1051-1060 (2000).

2. Goldberg, J. B. 'Pseudomonas '99, The Seventh International Congress on Pseudomonas: biotechnology and pathogenesis', organized by the American Society for Microbiology, was held in Maui, HI, USA, 1-5 September 1999. Trends Microbiol. 8, 55-57 (2000).

3. Wu, W. \& Jin, S. PtrB of Pseudomonas aeruginosa suppresses the type III secretion system under the stress of DNA damage. J. Bacteriol. 187, 6058-6068 (2005). 
4. Silby, M. W., Winstanley, C., Godfrey, S. A. C., Levy, S. B. \& Jackson, R. W. Pseudomonas genomes: Diverse and adaptable. FEMS Microbiol. Rev. 35, 652-680 (2011).

5. Molina-Mora, J.-A., Campos-Sánchez, R., Rodríguez, C., Shi, L. \& García, F. High quality 3C de novo assembly and annotation of a multidrug resistant ST-111 Pseudomonas aeruginosa genome: Benchmark of hybrid and non-hybrid assemblers. Sci. Rep. 10, $1392(2020)$.

6. Toval, F. et al. Predominance of carbapenem-resistant Pseudomonas aeruginosa isolates carrying blaIMP and blaVIM metallo$\beta$-lactamases in a major hospital in Costa Rica. J. Med. Microbiol. 64, 37-43 (2015).

7. Mulet, X. et al. Biological markers of Pseudomonas aeruginosa epidemic high-risk clones. Antimicrob. Agents Chemother. 57, 5527-5535 (2013).

8. World Health Organization. Guidelines for the prevention and control of carbapenem-resistant Enterobacteriaceae, Acinetobacter baumannii and Pseudomonas aeruginosa in health care facilities. (2017).

9. Woodford, N., Turton, J. F. \& Livermore, D. M. Multiresistant Gram-negative bacteria: The role of high-risk clones in the dissemination of antibiotic resistance. FEMS Microbiol. Rev. 35, 736-755 (2011).

10. Farajzadeh Sheikh, A. et al. Molecular epidemiology of colistin-resistant Pseudomonas aeruginosa producing NDM-1 from hospitalized patients in Iran. Iran. J. Basic Med. Sci. 22, 38-42 (2019).

11. Firme, M., Kular, H., Lee, C. \& Song, D. RpoS contributes to variations in the survival pattern of Pseudomonas aeruginosa in response to ciprofloxacin. J. Exp. Microbiol. Immunol. 14, 21-27 (2010).

12. Brazas, M. D., Brazas, M. D., Hancock, R. E. W. \& Hancock, R. E. W. Ciprofloxacin induction of a susceptibility determinant in Pseudomonas aeruginosa. Antimicrob. Agents Chemother. 49, 3222-3227 (2005).

13. McVicker, G. et al. Clonal expansion during Staphylococcus aureus infection dynamics reveals the effect of antibiotic intervention. PLoS Pathog. 10, 2 (2014).

14. Andersson, D. I. \& Hughes, D. Microbiological effects of sublethal levels of antibiotics. Nat. Rev. Microbiol. 12, 465-478 (2014).

15. Stewart, P. S. et al. Contribution of stress responses to antibiotic tolerance in Pseudomonas aeruginosa biofilms. Antimicrob. Agents Chemother. 59, 3838-3847 (2015).

16. Matern, W. M., Rifat, D., Bader, J. S. \& Karakousis, P. C. Gene enrichment analysis reveals major regulators of Mycobacterium tuberculosis gene expression in two models of antibiotic tolerance. Front. Microbiol. 9, 1-10 (2018).

17. Hocquet, D. et al. Evidence for induction of integron-based antibiotic resistance by the SOS response in a clinical setting. PLoS Pathog. 8, 2 (2012).

18. Dapa, T., Fleurier, S., Bredeche, M.-F. \& Matic, I. The SOS and RpoS regulons contribute to bacterial cell robustness to genotoxic stress by synergistically regulating DNA polymerase Pol II. Genetics 206, 1349-1360 (2017).

19. Kreuzer, K. N. DNA damage responses in prokaryotes: Regulating gene expression, modulating growth patterns, and manipulating replication forks. Cold Spring Harbor Perspect. Biol. https://doi.org/10.1101/cshperspect.a012674 (2013).

20. Valencia, E. Y., Esposito, F., Spira, B., Blázquez, J. \& Galhardo, R. S. Ciprofloxacin-mediated mutagenesis is suppressed by subinhibitory concentrations of amikacin in Pseudomonas aeruginosa. Antimicrob. Agents Chemother. AAC https://doi.org/10.1128/ AAC.02107-16 (2016).

21. Siqueira, V. L. D. et al. Structural changes and differentially expressed genes in Pseudomonas aeruginosa exposed to meropenemciprofloxacin combination. Antimicrob. Agents Chemother. 58, 3957-3967 (2014).

22. Cabot, G. et al. Evolution of Pseudomonas aeruginosa antimicrobial resistance and fitness under low and high mutation rates. Antimicrob. Agents Chemother. 60, 1767-1778 (2016).

23. Knezevic, P., Curcin, S., Aleksic, V., Petrusic, M. \& Vlaski, L. Phage-antibiotic synergism: A possible approach to combatting Pseudomonas aeruginosa. Res. Microbiol. 164, 55-60 (2013).

24. Dörr, T., Lewis, K. \& Vulić, M. SOS Response induces persistence to fluoroquinolones in Escherichia coli. PLoS Genet. 5, e1000760 (2009).

25. Recacha, E. et al. Quinolone resistance reversion by targeting the SOS response. MBio 8, 2 (2017).

26. Cirz, R. T., O'Neill, B. M., Hammond, J. A., Head, S. R. \& Romesberg, F. E. Defining the Pseudomonas aeruginosa SOS response and its role in the global response to the antibiotic ciprofloxacin. J. Bacteriol. 188, 7101-7110 (2006).

27. Breidenstein, E. B. M., Bains, M. \& Hancock, R. E. W. Involvement of the lon protease in the SOS response triggered by ciprofloxacin in Peudomonas aeruginosa PAO1. Antimicrob. Agents Chemother. 56, 2879-2887 (2012).

28. Shiba, T., Tsutsumi, K., Ishige, K. \& Noguchi, T. Inorganic polyphosphate and polyphosphate kinase: Their novel biological functions and applications. Biochem. 65, 315-323 (2000).

29. Suh, S. J. et al. Effect of rpoS mutation on the stress response and expression of virulence factors in Pseudomonas aeruginosa. J. Bacteriol. 181, 3890-3897 (1999).

30. Weber, H. et al. Genome-wide analysis of the general stress response network in Escherichia coli: sigmaS-dependent genes, promoters, and sigma factor selectivity. Society 187, 1591-1603 (2005).

31. Kayama, S. et al. The role of rpoS gene and quorum-sensing system in ofloxacin tolerance in Pseudomonas aeruginosa. FEMS Microbiol. Lett. 298, 184-192 (2009).

32. Hong, S. H., Wang, X., O’Connor, H. F., Benedik, M. J. \& Wood, T. K. Bacterial persistence increases as environmental fitness decreases. Microb. Biotechnol. 5, 509-522 (2012).

33. Baharoglu, Z. \& Mazel, D. SOS the formidable strategy of bacteria against aggressions. FEMS Microbiol. Rev. 38, 2 (2014).

34. Balasubramanian, D. et al. The regulatory repertoire of pseudomonas aeruginosa AmpC B-lactamase regulator AmpR includes virulence genes. PLoS ONE 7, 2 (2012).

35. Nguyen, H. et al. Negative control of RpoS synthesis by the sRNA ReaL in Pseudomonas aeruginosa. Front. Microbiol. 9, 1-10 (2018).

36. Müller, A. U., Imkamp, F. \& Weber-Ban, E. The mycobacterial LexA/RecA-independent DNA damage response is controlled by PafBC and the pup-proteasome system. Cell Rep. 23, 3551-3564 (2018).

37. Schulz, S. et al. Elucidation of sigma factor-associated networks in Pseudomonas aeruginosa reveals a modular architecture with limited and function-specific crosstalk. PLoS Pathog. 11, 1-21 (2015).

38. van Dam, S., Võsa, U., van der Graaf, A., Franke, L. \& de Magalhães, J. P. Gene co-expression analysis for functional classification and gene-disease predictions. Brief. Bioinform. 19, 575-592 (2018).

39. Linde, J., Schulze, S., Henkel, S. G. \& Guthke, R. Data- and knowledge-based modeling of gene regulatory networks: An update. EXCLI J. 14, 346-378 (2015).

40. Liu, W. et al. Construction and analysis of gene co-expression networks in Escherichia coli. Cells 7, 19 (2018).

41. Khaledi, A. et al. Transcriptome profiling of antimicrobial resistance in Pseudomonas aeruginosa. Antimicrob. Agents Chemother. 60, 4722-4733 (2016).

42. Fang, G. et al. Transcriptomic and phylogenetic analysis of a bacterial cell cycle reveals strong associations between gene coexpression and evolution. BMC Genom. 14, 2 (2013).

43. Langfelder, P. \& Horvath, S. WGCNA: An R package for weighted correlation network analysis. BMC Bioinform. 9, 2 (2008).

44. Lovelace, A. H., Smith, A. \& Kvitko, B. H. Pattern-triggered immunity alters the transcriptional regulation of virulence-associated genes and induces the sulfur starvation response in pseudomonas syringae pv. tomato DC3000. Mol. Plant-Microbe Interact. 31, $750-765$ (2018). 
45. Dai, H., Zhou, J. \& Zhu, B. Gene co-expression network analysis identifies the hub genes associated with immune functions for nocturnal hemodialysis in patients with end-stage renal disease. Med. (United States) 97, 1-8 (2018).

46. Chan, K.-G. et al. Transcriptome analysis of Pseudomonas aeruginosa PAO1 grown at both body and elevated temperatures. PeerJ 4, e2223 (2016).

47. Anupama, R., Sajitha Lulu, S., Mukherjee, A. \& Babu, S. Cross-regulatory network in Pseudomonas aeruginosa biofilm genes and $\mathrm{TiO} 2$ anatase induced molecular perturbations in key proteins unraveled by a systems biology approach. Gene 647, 289-296 (2018).

48. Molina-Mora, J. A., Campos-Sanchez, R. \& Garcia, F. Gene Expression Dynamics Induced by Ciprofloxacin and Loss of Lexa Function in Pseudomonas aeruginosa PAO1 Using Data Mining and Network Analysis. in 2018 IEEE International Work Conference on Bioinspired Intelligence (IWOBI) 1-7 (IEEE, 2018). doi: 10.1109/IWOBI.2018.8464130

49. Stojakovic, A., Mastronardi, C. A., Licinio, J. \& Wong, M.-L. Long-term consumption of high-fat diet impairs motor coordination without affecting the general motor activity. J. Transl. Sci. 5, 1-10 (2018).

50. Bjursell, M. et al. Ageing Fxr deficient mice develop increased energy expenditure, improved glucose control and liver damage resembling NASH. PLoS ONE 8, 2 (2013).

51. Bolger, A. M., Lohse, M. \& Usadel, B. Trimmomatic: a flexible trimmer for Illumina sequence data. Bioinformatics 30, 2114-2120 (2014).

52. Andrews, S. FastQC a quality control tool for high throughput sequence data. (2010). Available at: https://www.bioinformatics. babraham.ac.uk/projects/fastqc/. (Accessed: 10th April 2018)

53. Wingett, S. W. \& Andrews, S. FastQ Screen: A tool for multi-genome mapping and quality control. F1000Research 7, 1338 (2018).

54. Ewels, P., Magnusson, M., Lundin, S. \& Käller, M. MultiQC: summarize analysis results for multiple tools and samples in a single report. Bioinformatics 32, 3047-3048 (2016).

55. Magoc, T., Wood, D. \& Salzberg, S. L. EDGE-pro: estimated degree of gene expression in prokaryotic genomes. Evol. Bioinform. Online 9, 127-136 (2013).

56. Langmead, B. \& Salzberg, S. L. Fast gapped-read alignment with Bowtie 2. Nat. Methods 9, 357-359 (2012).

57. Okonechnikov, K., Conesa, A. \& García-Alcalde, F. Qualimap 2: Advanced multi-sample quality control for high-throughput sequencing data. Bioinformatics 32, 292-294 (2016).

58. Wang, L., Wang, S. \& Li, W. RSeQC: Quality control of RNA-seq experiments. Bioinformatics 28, 2184-2185 (2012).

59. Love, M. I., Huber, W. \& Anders, S. Moderated estimation of fold change and dispersion for RNA-seq data with DESeq2. Genome Biol. 15, 550 (2014).

60. R Core Team. R: A language and environment for statistical computing. R Foundation for Statistical Computing, Vienna, Austria. (2020).

61. Winsor, G. L. et al. Enhanced annotations and features for comparing thousands of Pseudomonas genomes in the Pseudomonas genome database. Nucleic Acids Res. 44, D646-D653 (2016).

62. Szklarczyk, D. et al. STRING v11: Protein-protein association networks with increased coverage, supporting functional discovery in genome-wide experimental datasets. Nucleic Acids Res. 47, D607-D613 (2019).

63. Shannon, P. et al. Cytoscape: A software environment for integrated models of biomolecular interaction networks. Genome Res. 13, 2498-2504 (2003).

64. Mine, A. et al. The defense phytohormone signaling network enables rapid, high-amplitude transcriptional reprogramming during effector-triggered immunity[OPEN]. Plant Cell 30, 1199-1219 (2018).

65. Wang, X. et al. Weighted gene co-expression network analysis for identifying hub genes in association with prognosis in Wilms tumor. Mol. Med. Rep. 19, 2041-2050 (2019).

66. Cao, L. et al. Identification of hub genes and potential molecular mechanisms in gastric cancer by integrated bioinformatics analysis. PeerJ 6, e5180 (2018).

67. Chin, C.-H. et al. cytoHubba: identifying hub objects and sub-networks from complex interactome. BMC Syst. Biol. 8, S11 (2014).

68. Teitzel, G. M. M. et al. Survival and growth in the presence of elevated copper: Transcriptional profiling of copper-stressed Pseudomonas aeruginosa. J. Bacteriol. 188, 7242-7256 (2006).

69. Cummins, J., Reen, F. J., Baysse, C., Mooij, M. J. \& O'Gara, F. Subinhibitory concentrations of the cationic antimicrobial peptide colistin induce the pseudomonas quinolone signal in Pseudomonas aeruginosa. Microbiology 155, 2826-2837 (2009).

70. Kai, T. et al. A low concentration of azithromycin inhibits the mRNA expression of $\mathrm{N}$-acyl homoserine lactone synthesis enzymes, upstream of lasI or rhlI, Pseudomonas aeruginosa. Pulm. Pharmacol. Ther. 22, 483-486 (2009).

71. Chang, W., Small, D. A., Toghrol, F. \& Bentley, W. E. Microarray analysis of Pseudomonas aeruginosa reveals induction of pyocin genes in response to hydrogen peroxide. BMC Genom. 6, 1-14 (2005).

72. Ceyssens, P.-J. Isolation and characterization of lytic bacteriophages infecting Pseudomonas aeruginosa (Katholieke Universiteit Leuven, Flanders, 2009).

73. Schwab, K. J., De Leon, R. \& Sobsey, M. D. Concentration and purification of beef extract mock eluates from water samples for the detection of enteroviruses, hepatitis A virus, and Norwalk virus by reverse transcription-PCR. Appl. Environ. Microbiol. 61, 531-537 (1995)

74. Paterson, W. D., Douglas, R. J., Grinyer, I. \& McDermott, L. A. Isolation and preliminary characterization of some Aeromonas salmonicida bacteriophages. J. Fish. Res. Board Canada 26, 629-632 (1969).

75. Schuster, M., Hawkins, A. C., Harwood, C. S. \& Greenberg, E. P. The Pseudomonas aeruginosa RpoS regulon and its relationship to quorum sensing. Mol. Microbiol. 51, 973-985 (2004).

76. Kumar, S. S., Penesyan, A., Elbourne, L. D. H., Gillings, M. R. \& Paulsen, I. T. Catabolism of Nucleic acids by a cystic fibrosis Pseudomonas aeruginosa isolate: An adaptive pathway to cystic fibrosis sputum environment. Front. Microbiol. 10, 1-14 (2019).

77. Fernández, M., Corral-Lugo, A. \& Krell, T. The plant compound rosmarinic acid induces a broad quorum sensing response in Pseudomonas aeruginosa PAO1. Environ. Microbiol. 20, 4230-4244 (2018).

78. Salmon-Divon, M., Zahavi, T. \& Kornspan, D. Transcriptomic analysis of the brucella melitensisrev.1 vaccine strain in an acidic environment: Insights into virulence attenuation. Front. Microbiol. 10, 1-12 (2019).

79. Thode, S. K. et al. Construction of a fur null mutant and RNA-sequencing provide deeper global understanding of the Alivibrio salmonicida Fur regulon. PeerJ 2017, 2 (2017).

80. Mets, T. et al. Fragmentation of Escherichia coli mRNA by MazF and MqsR. Biochimie 156, 79-91 (2019).

81. Cabezas, C. E. et al. The transcription factor SlyA from Salmonella Typhimurium regulates genes in response to hydrogen peroxide and sodium hypochlorite. Res. Microbiol. 169, 263-278 (2018).

82. Fornelos, N., Browning, D. F. \& Butala, M. The use and abuse of LexA by mobile genetic elements. Trends Microbiol. 24, 391-401 (2016).

83. Stockwell, V. O. \& Loper, J. E. The sigma factor RpoS is required for stress tolerance and environmental fitness of Pseudomonas fluorescens Pf-5. Microbiology 151, 3001-3009 (2005).

84. Goerke, C., Koller, J. \& Wolz, C. Ciprofloxacin and trimethoprim cause phage induction and virulence modulation in Staphylococcus aureus. Antimicrob. Agents Chemother. 50, 171-177 (2006).

85. Friedman, N., Vardi, S., Ronen, M., Alon, U. \& Stavans, J. Precise temporal modulation in the response of the SOS DNA repair network in individual bacteria. PLoS Biol. 3, e238 (2005). 
86. Ronen, M., Rosenberg, R., Shraiman, B. I. \& Alon, U. Assigning numbers to the arrows: Parameterizing a gene regulation network by using accurate expression kinetics. Proc. Natl. Acad. Sci. USA. 99, 10555-10560 (2002).

87. Babin, B. M. et al. Selective proteomic analysis of antibiotic-tolerant cellular subpopulations in pseudomonas aeruginosa biofilms. MBio 8, 2 (2017).

88. Kamal, F. \& Dennis, J. J. Burkholderia cepacia complex phage-antibiotic synergy (PAS): Antibiotics stimulate lytic phage activity. Appl. Environ. Microbiol. 81, 1132-1138 (2015).

89. Burmeister, A. R. et al. Pleiotropy complicates a trade-off between phage resistance and antibiotic resistance. Proc. Natl. Acad. Sci. USA. https://doi.org/10.1073/pnas.1919888117 (2020).

90. Ryan, E. M., Alkawareek, M. Y., Donnelly, R. F. \& Gilmore, B. F. Synergistic phage-antibiotic combinations for the control of Escherichia coli biofilms in vitro. FEMS Immunol. Med. Microbiol. 65, 395-398 (2012).

91. Golais, F., Hollý, J. \& Vítkovská, J. Coevolution of bacteria and their viruses. Folia Microbiol. (Praha) 58, 177-186 (2013).

92. Fothergill, J. L. et al. Effect of antibiotic treatment on bacteriophage production by a cystic fibrosis epidemic strain of Pseudomonas aeruginosa. Antimicrob. Agents Chemother. 55, 426-428 (2011).

93. Chatterjee, M. et al. Antibiotic resistance in Pseudomonas aeruginosa and alternative therapeutic options. Int. J. Med. Microbiol. 306, 48-58 (2016).

94. Rozanov, D. V., D’Ari, R. \& Sineoky, S. P. RecA-independent pathways of lambdoid prophage induction in Escherichia coli. J. Bacteriol. 180, 6306-6315 (1998).

95. Xie, X. T. Characterization of the fecal virome and fecal virus shedding patterns of commercial mink (Neovison vison) (University of Guelph, Guelph, 2017).

96. Tsao, Y. F. et al. Phage morons play an important role in Pseudomonas aeruginosa phenotypes. J. Bacteriol. 200, 1-15 (2018).

97. Winstanley, C. et al. Newly introduced genomic prophage islands are critical determinants of in vivo competitiveness in the Liverpool epidemic strain of Pseudomonas aeruginosa. Genome Res. 19, 12-23 (2008).

98. Blasdel, B. G., Chevallereau, A., Monot, M., Lavigne, R. \& Debarbieux, L. Comparative transcriptomics analyses reveal the conservation of an ancestral infectious strategy in two bacteriophage genera. ISME J. 11, 1988-1996 (2017).

99. Chevallereau, A. et al. Next-generation-omics approaches reveal a massive alteration of host RNA metabolism during bacteriophage infection of Pseudomonas aeruginosa. PLoS Genet. 12, 1-20 (2016).

100. De Smet, J. et al. High coverage metabolomics analysis reveals phage-specific alterations to Pseudomonas aeruginosa physiology during infection. ISME J. 10, 1823-1835 (2016).

101. Podkovyrov, S. \& Larson, T. J. Lipid biosynthetic genes and a ribosomal protein gene are cotranscribed. FEBS Lett. 368, 429-431 (1995).

102. Liu, S. et al. Identification of novel genes including rpmF and yjjQ critical for Type II 1 persister formation in Escherichia coli. bioRxiv https://doi.org/10.1101/310961 (2018).

103. Cornforth, D. M. et al. Pseudomonas aeruginosa transcriptome during human infection. Proc. Natl. Acad. Sci. U. S. A. 115, 2 (2018).

104. Quintana, J., Novoa-Aponte, L. \& Argüello, J. M. Copper homeostasis networks in the bacterium Pseudomonas aeruginosa. J. Biol. Chem. 292, 15691-15704 (2017).

105. Zheng, X., Su, Y., Chen, Y., Huang, H. \& Shen, Q. Global transcriptional responses of denitrifying bacteria to functionalized single-walled carbon nanotubes revealed by weighted gene-coexpression network analysis. Sci. Total Environ. 613-614, $1240-1249$ (2018).

106. Shin, H., Jeon, J., Lee, J.-H., Jin, S. \& Ha, U.-H. Pseudomonas aeruginosa GroEL stimulates production of PTX3 by activating the NF-kB pathway and simultaneously downregulating MicroRNA-9. Infect. Immun. 85, 2 (2017).

107. Ito, F., Tamiya, T., Ohtsu, I., Fujimura, M. \& Fukumori, F. Genetic and phenotypic characterization of the heat shock response in Pseudomonas putida. Microbiologyopen 3, 922-936 (2014).

108. Michta, E. et al. Proteomic approach to reveal the regulatory function of aconitase AcnA in oxidative stress response in the antibiotic producer Streptomyces viridochromogenes Tü494. PLoS ONE 9, 1 (2014).

109. Wang, Y., Kern, S. E. \& Newman, D. K. Endogenous phenazine antibiotics promote anaerobic survival of Pseudomonas aeruginosa via extracellular electron transfer. J. Bacteriol. 192, 365-369 (2010).

110. Higgins, S. et al. Differential regulation of the phenazine biosynthetic operons by quorum sensing in Pseudomonas aeruginosa PAO1-N. Front. Cell. Infect. Microbiol. 8, 2 (2018).

\section{Acknowledgements}

We thank the students John Rodríguez Fernández and Daniel Ulate Rodríguez for their collaboration in multiple activities of the project. We also thank the Genome Technology Center of New York University for generating all sequencing data used in this work, and to all members of Centro de Investigación en Enfermedades Tropicales (Universidad de Costa, Costa Rica) for their support in the experimental assays, and PGx group of The Human Phenome Institute (Fudan University, Shanghai-China) for their support in the bioinformatic analysis. Finally we thank Rachel O'Dea for the critical reading of this work. This work was funded by project "B8114 Definición de la red transcriptómica y de las alteraciones genómicas inducidas por ciprofloxacina en Pseudomonas aeruginosa AG1", Vicerrectoría de Investigación, Universidad de Costa Rica (period 2017-2019).

\section{Author contributions}

J.M.M. and F.G. participated in the conception, design of the study and data selection. D.C.M., M.C.A. and A.U.M. run the experimental assays. J.M.M. implemented all bioinformatic analysis. J.M.M., R.C.S., R.M.R. and L.S. were involved in bioinformatic analysis interpretation. J.M.M. and F.G. participated in the interpretation of the data in the biological context. J.M.M. drafted the manuscript and all authors were involved in its revision. All authors read and approved the final manuscript.

\section{Competing interests}

The authors declare no competing interests.

\section{Additional information}

Supplementary information is available for this paper at https://doi.org/10.1038/s41598-020-70581-2.

Correspondence and requests for materials should be addressed to J.A.M.-M.

Reprints and permissions information is available at www.nature.com/reprints. 
Publisher's note Springer Nature remains neutral with regard to jurisdictional claims in published maps and institutional affiliations.

(c) (i) Open Access This article is licensed under a Creative Commons Attribution 4.0 International License, which permits use, sharing, adaptation, distribution and reproduction in any medium or format, as long as you give appropriate credit to the original author(s) and the source, provide a link to the Creative Commons license, and indicate if changes were made. The images or other third party material in this article are included in the article's Creative Commons license, unless indicated otherwise in a credit line to the material. If material is not included in the article's Creative Commons license and your intended use is not permitted by statutory regulation or exceeds the permitted use, you will need to obtain permission directly from the copyright holder. To view a copy of this license, visit http://creativecommons.org/licenses/by/4.0/.

(c) The Author(s) 2020 